\title{
GROMOV HYPERBOLICITY OF DENJOY DOMAINS
}

\author{
VENANCIO ALVAREZ, ANA PORTILLA(1), JOSE M. RODRIGUEZ (1)(2) AND EVA TOURIS(1)(3)
}

\begin{abstract}
In this paper we characterize the Gromov hyperbolicity of the double of a metric space. This result allows to give a characterization of the hyperbolic Denjoy domains, in terms of the distance to $\mathbb{R}$ of the points in some geodesics. In the particular case of trains (a kind of Riemann surfaces which includes the flute surfaces), we obtain more explicit criteria which depend just on the lengths of what we have called fundamental geodesics.
\end{abstract}

Key words and phrases: Denjoy domain, flute surface, Gromov hyperbolicity, Riemann surface, Schottky double, train.

\section{INTRODUCTION.}

To understand the connections between graphs and Potential Theory on Riemannian manifolds (see e.g. [4], [14], [20], [25], [26], [27], [35]), Gromov hyperbolic spaces are a useful tool. Besides, the concept of Gromov hyperbolicity grasps the essence of negatively curved spaces, and has been successfully used in the theory of groups (see e.g. [22] and the references therein).

A geodesic metric space is called hyperbolic (in the Gromov sense) if there is an upper bound of the distance of every point in a side of any geodesic triangle to the union of the two other sides (see Definition 2.3). The condition above is due to Rips.

But, it is not easy to determine if a given space is Gromov hyperbolic or not. One interesting instance is that of a Riemann surface endowed with the Poincaré metric. With that metric structure a Riemann surface is negatively curved, but not all Riemann surfaces are Gromov hyperbolic, since topological obstacles may impede it: for instance, the two-dimensional jungle-gym (a $\mathbb{Z}^{2}$-covering of a torus with genus two) is not hyperbolic.

We are interested in studying when Riemann surfaces equipped with their Poincaré metric are Gromov hyperbolic ([30], [31], [32]). To be more precise, in this paper our main aim is to study the hyperbolicity of Denjoy domains, that is to say, plane domains $\Omega$ with $\partial \Omega \subset \mathbb{R}$. This kind of surfaces are becoming more and more important in Geometric Theory of Functions, since, on the one hand, they are a very general type of Riemann surfaces, and, on the other hand, their symmetry simplifies their study. However, our techniques let us get as well several characterizations for a more general kind of space: the Schottky double of a Riemann surface, and even the double of a metric space (see Theorem 3.2). This result gives several characterizations of hyperbolic Denjoy domains (see Theorem 5.1), since every Denjoy domain is also a Schottky double.

One of these characterizations is particularly surprising: it is sufficient to check the Rips condition only on geodesic "bigons" (triangles with two vertices); this is clearly false in the general case: every geodesic bigon in $\mathbb{R}^{n}$ (with the euclidean distance) is 0 -thin, but $\mathbb{R}^{n}$ is not hyperbolic if $n>1$. So, in general, it is necessary to check the Rips condition for all triangles.

Our main characterization gives that a Denjoy domain is hyperbolic if and only if the distance to $\mathbb{R}$ of any point in any simple closed geodesic is uniformly bounded.

Date: May 4, 2005.

2000 AMS Subject Classification: 41A10, 46E35, 46G10.

(1) Research partially supported by a grant from DGI (BFM 2003-04870), Spain.

(2) Research partially supported by a grant from DGI (BFM 2003-06335-C03-02), Spain.

(3) Research partially supported by a grant from DGI (BFM 2000-0022), Spain. 
Nevertheless, Denjoy domains are such a wide class of Riemann surfaces that characterization criteria are not straightforward to apply. That is the main reason because of we decided to focus on two particular types of Denjoy domains, which we have called trains (see Definition 5.2) and generalized trains (see Definition 5.29). About them, we have been able to obtain both characterizations and sufficient conditions that either guarantee or discard hyperbolicity.

We study the hyperbolicity of trains in terms of the lengths of two types of their simple closed geodesics, which we have named as fundamental (see Definition 5.2), and whose lengths are denoted by $l_{n}$ and $r_{n}$. So, for instance, Theorem 5.3 provides a characterization of the hyperbolicity of trains which does not require any other condition.

One of the major novelties of this paper is that most of the hyperbolicity criteria depend on the fundamental geodesics just through their lengths $l_{n}$ and $r_{n}$.

The approximation to the problem of the hyperbolicity of trains requires different strategies according to the behavior of the sequences $\left\{l_{n}\right\}_{n}$ and $\left\{r_{n}\right\}_{n}$. So:

1. If $\left\{l_{n}\right\}_{n}$ is bounded, the train is always hyperbolic, regardless of what happens with $\left\{r_{n}\right\}_{n}$ (see Theorem 5.25).

2. If $\left\{l_{n}\right\}_{n}$ is not bounded, in general we are going to require that $\left\{r_{n}\right\}_{n}$ is bounded in order to guarantee hyperbolicity. In fact, Theorem 5.17 and corollaries 5.18 and 5.19 discard hyperbolicity in most cases when $\left\{r_{n}\right\}_{n}$ is not bounded.

2.1. If $\lim _{n \rightarrow \infty} l_{n}=\infty$, Theorem 5.14 is a characterization of hyperbolicity and theorems $5.12,5.21$ and 5.24 provide sufficient conditions.

2.2. Otherwise, we have both a characterization of hyperbolicity (see Theorem 5.26) and a sufficient condition (see Theorem 5.27).

Theorems 5.30 and 5.31 are characterizations for generalized trains. And finally, Theorem 5.33 is a result about stability of hyperbolicity under bounded perturbations of the lengths of the fundamental geodesics, even though the original surface and the modified one are not quasi-isometric.

These results let us get interesting examples of hyperbolic and non-hyperbolic Riemann surfaces.

Notations. We denote by $X$ a geodesic metric space. By $d_{X}$ and $L_{X}$ we shall denote, respectively, the distance and the length in the metric of $X$. From now on, when there is no possible confusion, we will not write the subindex $X$.

We denote by $\Omega$ a Denjoy domain with its Poincaré metric.

We denote by $\Re z$ and $\Im z$ the real and imaginary part of $z$, respectively.

Finally, we denote by $c$ and $c_{i}$, positive constants which can assume different values in different theorems.

Acknowledgements. We would like to thank Professor J. L. Fernández for some useful discussions.

\section{Background in Gromov spaces.}

In our study of hyperbolic Gromov spaces we use the notations of [22]. We give now the basic facts about these spaces. We refer to [22] for more background and further results.

Definition 2.1. Let us fix a point $w$ in a metric space $(X, d)$. We define the Gromov product of $x, y \in X$ with respect to the point $w$ as

$$
(x \mid y)_{w}:=\frac{1}{2}(d(x, w)+d(y, w)-d(x, y)) \geq 0 .
$$

We say that the metric space $(X, d)$ is $\delta$-hyperbolic $(\delta \geq 0)$ if

$$
(x \mid z)_{w} \geq \min \left\{(x \mid y)_{w},(y \mid z)_{w}\right\}-\delta,
$$

for every $x, y, z, w \in X$. We say that $X$ is hyperbolic (in the Gromov sense) if the value of $\delta$ is not important. 
It is convenient to remark that this definition of hyperbolicity is not universally accepted, since sometimes the word hyperbolic refers to negative curvature or to the existence of Green's function. However, in this paper we only use the word hyperbolic in the sense of Definition 2.1.

\section{Examples:}

(1) Every bounded metric space $X$ is (diam $X$ )-hyperbolic (see e.g. [22], p.29).

(2) Every complete simply connected Riemannian manifold with sectional curvature which is bounded from above by $-k$, with $k>0$, is hyperbolic (see e.g. [22], p.52).

(3) Every tree with edges of arbitrary length is 0-hyperbolic (see e.g. [22], p.29).

Definition 2.2. If $\gamma:[a, b] \longrightarrow X$ is a continuous curve in a metric space $(X, d)$, we can define the length of $\gamma$ as

$$
L(\gamma):=\sup \left\{\sum_{i=1}^{n} d\left(\gamma\left(t_{i-1}\right), \gamma\left(t_{i}\right)\right): a=t_{0}<t_{1}<\cdots<t_{n}=b\right\} .
$$

We say that $\gamma$ is a geodesic if it is an isometry, i.e. $L\left(\left.\gamma\right|_{[t, s]}\right)=d(\gamma(t), \gamma(s))=|t-s|$ for every $s, t \in[a, b]$. We say that $X$ is a geodesic metric space if for every $x, y \in X$ there exists a geodesic joining $x$ and $y$; we denote by $[x, y]$ any of such geodesics (since we do not require uniqueness of geodesics, this notation is ambiguous, but it is convenient). It is clear that every geodesic metric space is path-connected.

Definition 2.3. If $X$ is a geodesic metric space and $J$ is a polygon whose sides are $J_{1}, J_{2}, \ldots, J_{n}$, we say that $J$ is $\delta$-thin if for every $x \in J_{i}$ we have that $d\left(x, \cup_{j \neq i} J_{j}\right) \leq \delta$. If $x_{1}, x_{2}, x_{3} \in X$, a geodesic triangle $T=\left\{x_{1}, x_{2}, x_{3}\right\}$ is the union of three geodesics $\left[x_{1}, x_{2}\right],\left[x_{2}, x_{3}\right]$ and $\left[x_{3}, x_{1}\right]$. The space $X$ is $\delta$-thin (or satisfies the Rips condition with constant $\delta$ ) if every geodesic triangle in $X$ is $\delta$-thin.

If we have a triangle with two identical vertices, we call it a "bigon". Obviously, every bigon in a $\delta$-thin space is $\delta$-thin. It is also clear that every geodesic polygon with $n$ sides in a $\delta$-thin space is $(n-2) \delta$-thin.

Definition 2.4. Given a geodesic triangle $T=\{x, y, z\}$ in a geodesic metric space $X$, let $T_{E}$ be a Euclidean triangle with sides of the same length than $T$. Since there is no possible confusion, we will use the same notation for the corresponding points in $T$ and $T_{E}$. The maximum inscribed circle in $T_{E}$ meets the side $[x, y]$ (respectively $[y, z],[z, x])$ in a point $z^{\prime}$ (respectively $\left.x^{\prime}, y^{\prime}\right)$ such that $d\left(x, z^{\prime}\right)=d\left(x, y^{\prime}\right), d\left(y, x^{\prime}\right)=d\left(y, z^{\prime}\right)$ and $d\left(z, x^{\prime}\right)=d\left(z, y^{\prime}\right)$. We call the points $x^{\prime}, y^{\prime}, z^{\prime}$, the internal points of $\{x, y, z\}$. There is a unique isometry $f$ of the triangle $\{x, y, z\}$ onto a tripod (a tree with one vertex $w$ of degree 3 , and three vertices $x^{\prime \prime}, y^{\prime \prime}, z^{\prime \prime}$ of degree one, such that $d\left(x^{\prime \prime}, w\right)=d\left(x, z^{\prime}\right)=d\left(x, y^{\prime}\right), d\left(y^{\prime \prime}, w\right)=d\left(y, x^{\prime}\right)=d\left(y, z^{\prime}\right)$ and $\left.d\left(z^{\prime \prime}, w\right)=d\left(z, x^{\prime}\right)=d\left(z, y^{\prime}\right)\right)$. The triangle $\{x, y, z\}$ is $\delta$-fine if $f(p)=f(q)$ implies that $d(p, q) \leq \delta$. The space $X$ is $\delta$-fine if every geodesic triangle in $X$ is $\delta$-fine.

A basic result is that hyperbolicity is equivalent to Rips condition and to be fine:

Theorem 2.5. ([22], p.41) Let us consider a geodesic metric space $X$.

(1) If $X$ is $\delta$-hyperbolic, then it is $4 \delta$-thin and $4 \delta$-fine.

(2) If $X$ is $\delta$-thin, then it is $4 \delta$-hyperbolic and $4 \delta$-fine.

(3) If $X$ is $\delta$-fine, then it is $2 \delta$-hyperbolic and $\delta$-thin.

We present now the class of maps which play the main role in the theory.

Definition 2.6. A function between two metric spaces $f: X \longrightarrow Y$ is a quasi-isometry if there are constants $a \geq 1, b \geq 0$ with

$$
\frac{1}{a} d_{X}\left(x_{1}, x_{2}\right)-b \leq d_{Y}\left(f\left(x_{1}\right), f\left(x_{2}\right)\right) \leq a d_{X}\left(x_{1}, x_{2}\right)+b, \quad \text { for every } x_{1}, x_{2} \in X .
$$

A such function is called an $(a, b)$-quasi-isometry. We say that the image of $f$ is $\varepsilon$-full (for some $\varepsilon \geq 0)$ if for every $y \in Y$ there exists $x \in X$ with $d_{Y}(y, f(x)) \leq \varepsilon$. We say that $X$ and $Y$ are quasi-isometrically equivalent if there exists a quasi-isometry between $X$ and $Y$, with image $\varepsilon$-full, for some $\varepsilon \geq 0$. An $(a, b)$-quasigeodesic 
in $X$ is an $(a, b)$-quasi-isometry between an interval of $\mathbb{R}$ and $X$. An $(a, b)$-quasigeodesic segment in $X$ is an $(a, b)$-quasi-isometry between a compact interval of $\mathbb{R}$ and $X$.

Let us observe that a quasi-isometry can be discontinuous.

Remark. It is well known (see e.g. [26], [27]) that quasi-isometrical equivalence is an equivalence relation. In fact, if $f: X \longrightarrow Y$ is an $(a, b)$-quasi-isometry with image $\varepsilon$-full, then there exists a function $g: Y \longrightarrow X$ which is an $(a, 2 a \varepsilon+a b)$-quasi-isometry. In particular, if $f$ is a surjective $(a, b)$-quasi-isometry, then $g$ is an $(a, a b)$-quasi-isometry (in this case we can choose as $g(y)$ any point in $f^{-1}(y)$ ).

Quasi-isometries are important since they are the maps which preserve hyperbolicity:

Theorem 2.7. ([22], p.88) Let us consider an $(a, b)$-quasi-isometry between two geodesic metric spaces $f: X \longrightarrow Y$. If $Y$ is $\delta$-hyperbolic, then $X$ is $\delta^{\prime}$-hyperbolic, where $\delta^{\prime}$ is a constant which only depends on $\delta$, a and $b$. Besides, if the image of $f$ is $\varepsilon$-full for some $\varepsilon \geq 0$, then $X$ is hyperbolic if and only if $Y$ is hyperbolic.

It is well-known that if $f$ is not $\varepsilon$-full, the hyperbolicity of $X$ does not imply the hyperbolicity of $Y$ : it is enough to consider the inclusion of $\mathbb{R}$ in $\mathbb{R}^{2}$ (which is indeed an isometry).

Definition 2.8. Let us consider $H>0$, a metric space $X$, and subsets $Y, Z \subseteq X$. The set $V_{H}(Y):=\{x \in$ $X: d(x, Y) \leq H\}$ is called the $H$-neighborhood of $Y$ in $X$. The Hausdorff distance of $Y$ to $Z$ is defined by $\mathcal{H}(Y, Z):=\inf \left\{H>0: Y \subseteq V_{H}(Z), Z \subseteq V_{H}(Y)\right\}$.

The following is a beautiful and useful result:

Theorem 2.9. ([22], p.87) For each $\delta \geq 0, a \geq 1$ and $b \geq 0$, there exists a constant $H=H(\delta, a, b)$ with the following property:

Let us consider a $\delta$-hyperbolic geodesic metric space $X$ and an $(a, b)$-quasigeodesic $g$ starting in $x$ and finishing in $y$. If $\gamma$ is a geodesic joining $x$ and $y$, then $\mathcal{H}(g, \gamma) \leq H$.

This property is known as geodesic stability. Mario Bonk has proved that, in fact, geodesic stability is equivalent to hyperbolicity [9].

Along this paper we will work with topological subspaces of a geodesic metric space $X$. There is a natural way to define a distance in these spaces:

Definition 2.10. If $X$ is a path-connected space in which we have defined the length L of any curve, we can consider the intrinsic distance with respect to $L$

$$
d_{X}(x, y):=\inf \{L(\gamma): \gamma \subset X \text { is a continuous curve joining } x \text { and } y\} .
$$

In order to prove Theorem 3.2 below, we need the following elementary results (see e.g. [30], Lemma 2.16 and [30], Lemma 2.24 for some proofs):

Lemma 2.11. For each $\delta, b \geq 0$ and $a \geq 1$, there exists a constant $K=K(\delta, a, b)$ with the following property: If $X$ is a $\delta$-hyperbolic geodesic metric space and $T \subseteq X$ is an $(a, b)$-quasigeodesic triangle, then $T$ is $K$-thin. Furthermore, $K=4 \delta+2 H(\delta, a, b)$, where $H$ is the constant in Theorem 2.9.

Lemma 2.12. Let us consider a metric space $X$, an interval $I$, an $(a, b)$-quasigeodesic $g: I \longrightarrow X$ and a curve $g_{1}: I \longrightarrow X$ such that $d\left(g(t), g_{1}(t)\right) \leq c$ for every $t \in I$. Then $g_{1}$ is an $(a, b+2 c)$-quasigeodesic.

\section{Results in MEtRiC SPACES.}

Let us introduce now the kind of spaces which will be the main topic of the current paper.

Definition 3.1. Given a geodesic metric space $X$ and closed connected pairwise disjoint subsets $\left\{\eta_{j}\right\}_{j \in J}$ of $X$, we consider another copy $X^{\prime}$ of $X$. The double $D X$ of $X$ is the union of $X$ and $X^{\prime}$ obtained by identifying the corresponding points in each $\eta_{j}$ and $\eta_{j}^{\prime}$. 
Since $X$ and $X^{\prime}$ are metric spaces, we have defined the length $L$ of any curve. We always consider $D X$ with its intrinsic distance with respect to this $L$. If $X=S$ is a bordered surface and $\partial S=\cup_{j \in J} \eta_{j}, D S$ is known as the Schottky double of $S$ (see e.g. [1], p.119).

The following result gives several characterizations of the hyperbolicity of the double $D X$. These characterizations mean a new approach to the study of the hyperbolicity: now it is sufficient to bound the distance between some geodesics and $\cup_{j \in J} \eta_{j}$, and then the amount of geodesics to check is drastically reduced with respect to Rips condition.

Theorem 3.2. Let us consider a geodesic metric space $X$ and closed connected pairwise disjoint subsets $\left\{\eta_{j}\right\}_{j \in J}$ of $X$, such that the double DX is a geodesic metric space. Then the following conditions are equivalent:

(1) DX is $\delta$-hyperbolic.

(2) $X$ is $\delta_{0}$-hyperbolic and there exists a constant $c_{1}$ such that for every $k, l \in J$ and $a \in \eta_{k}, b \in \eta_{l}$ we have $d_{X}\left(x, \cup_{j \in J} \eta_{j}\right) \leq c_{1}$ for every $x \in[a, b] \subset X$.

(3) $X$ is $\delta_{0}$-hyperbolic and there exists a constant $c_{2}$ such that for every $k, l \in J$ and $a \in \eta_{k}, b \in \eta_{l}$ there exist $a_{0} \in \eta_{k}, b_{0} \in \eta_{l}$ with $d_{X}\left(x, \cup_{j \in J} \eta_{j}\right) \leq c_{2}$ for every $x \in\left[a, a_{0}\right] \cup\left[a_{0}, b_{0}\right] \cup\left[b, b_{0}\right] \subset X$.

(4) $X$ is $\delta_{0}$-hyperbolic and there exists a constant $c_{3}$ such that every geodesic bigon in $D X$ with vertices in $\cup_{j \in J} \eta_{j}$ is $c_{3}$-thin.

(5) $X$ is $\delta_{0}$-hyperbolic and there exist constants $c_{4}, \alpha, \beta$ such that for every $k, l \in J$ and $a \in \eta_{k}, b \in \eta_{l}$ we have $d_{X}\left(x, \cup_{j \in J} \eta_{j}\right) \leq c_{4}$ for every $x$ in some $(\alpha, \beta)$-quasigeodesic joining a with $b$ in $X$.

(6) $X$ is $\delta_{0}$-hyperbolic and there exist constants $c_{5}, \alpha, \beta$ such that for every $k, l \in J$ and $a \in \eta_{k}, b \in \eta_{l}$ there exist $a_{0} \in \eta_{k}, b_{0} \in \eta_{l}$ with $d_{X}\left(x, \cup_{j \in J} \eta_{j}\right) \leq c_{5}$ for every $x$ in some $(\alpha, \beta)$-quasigeodesic joining $a$ with $a_{0}$ in $X$ and some $(\alpha, \beta)$-quasigeodesic joining $b$ with $b_{0}$ in $X$, and $d_{X}\left(x, \cup_{j \in J} \eta_{j}\right) \leq c_{5}$ for every $x$ in some $(\alpha, \beta)$-quasigeodesic joining a with $b$ in $X$.

Furthermore, the constants in each condition only depend on the constants appearing in any other of the conditions.

Remark. By Theorem 2.9, by $[a, b],\left[a, a_{0}\right],\left[b, b_{0}\right]$ and $\left[a_{0}, b_{0}\right]$ in $(2)$ and (3) we can mean some particular choice of these geodesics.

Proof. We prove first that (1) implies (4). If $D X$ is $\delta$-hyperbolic, then $X$ is $\delta$-hyperbolic, since $X$ is geodesically convex in $D X$ (recall that $X^{\prime}$ is isometric to $X$ and that the intrinsic distance in $X$ given by $d_{D X}$ is equal to $\left.d_{X}\right)$. It is direct that every bigon is $4 \delta$-thin, by Theorem 2.5 .

Let us see that (4) implies (2). Consider $k, l \in J, a \in \eta_{k}, b \in \eta_{l}$ and $x \in[a, b] \subset X$. Let us denote by $[a, b]^{\prime}$ the symmetric geodesic in $D X$ of $[a, b]$. Since $[a, b] \cup[a, b]^{\prime}$ is a geodesic bigon in $D X$, we have $d_{D X}\left(x,[a, b]^{\prime}\right) \leq c_{3}$. Consequently, $d_{D X}\left(x, \cup_{j \in J} \eta_{j}\right) \leq c_{3}$, since $X \cap X^{\prime}=\cup_{j \in J} \eta_{j}$.

We prove now that (2) implies (1). Denote by $g$ the isometry of $D X$ which maps the points of $X$ in their symmetric points in $X^{\prime}$ (and viceversa). Let us consider a geodesic triangle $T=\{a, b, c\}$ in $D X$ and the triangle $T_{0}$ in $X$ obtained by changing in $T$ the set $T \cap X^{\prime}$ by $g\left(T \cap X^{\prime}\right)$.

If the three vertices are in $X$, let us observe that $d_{D X}(x, g(x)) \leq 2 c_{1}$ for every $x \in T \cap X^{\prime}$; it is clear that $T$ is a geodesic triangle $\left(4 \delta_{0}+4 c_{1}\right)$-thin, since $T_{0}$ is $4 \delta_{0}$-thin. In other case, we can assume by symmetry, that $a, b \in X$ and $c \in X^{\prime}$. The side in $T_{0}$ joining $a$ and $b$ is geodesic in $D X$. Let us denote by $a_{0}$ (respectively, $b_{0}$ ) the last point in $[a, c]$ (respectively, $[b, c]$ ) which belongs to $X$; it is clear that $a_{0}, b_{0} \in \cup_{j \in J} \eta_{j}$. The subsets $\left[a, a_{0}\right]$ and $\left[b, b_{0}\right]$ of $T_{0}$ are geodesics in $D X$. It is clear that $\left[a, a_{0}\right] \cup\left[a_{0}, c\right]$ and $\left[b, b_{0}\right] \cup\left[b_{0}, c\right]$ are also geodesics in $D X$.

We consider a geodesic triangle $T_{c}=\left\{a_{0}, b_{0}, c\right\}$ in $D X$ (contained in $X^{\prime}$ ) with $\left[a_{0}, c\right],\left[b_{0}, c\right] \subset T$. Let us denote by $c^{1}, b^{1}, a^{1}$ the internal points (see Definition 2.4) of $T_{c}$ in the geodesics $\left[a_{0}, b_{0}\right],\left[a_{0}, c\right],\left[c, b_{0}\right]$, respectively. We define $T_{1}$ as the (not necessarily geodesic) triangle with vertices $a, b, c^{1}$, obtained from $T_{0}$ by replacing $g\left(\left[a_{0}, c\right]\right) \cup g\left(\left[c, b_{0}\right]\right) \subset X$ by $\left[a_{0}, b_{0}\right]=\left[a_{0}, c^{1}\right] \cup\left[c^{1}, b_{0}\right] \subset X^{\prime}$. We have that $d_{D X}(x, g(x)) \leq 2 c_{1}$ for every $x \in\left[a_{0}, b_{0}\right]$. Let us observe that $L\left(\left[a_{0}, b^{1}\right]\right)=L\left(\left[a_{0}, c^{1}\right]\right)$ and $L\left(\left[b_{0}, a^{1}\right]\right)=L\left(\left[b_{0}, c^{1}\right]\right)$. Then, Lemma 
2.12 gives that $T_{1}$ is $\left(1,8 \delta_{0}\right)$-quasigeodesic in $D X$, since $X^{\prime}$ is $4 \delta_{0}$-fine. If $T_{2}:=g\left(T_{1}\right)$, hypothesis $(2)$ and Lemma 2.12 imply that $T_{2} \subset X$ is $\left(1,8 \delta_{0}+4 c_{1}\right)$-quasigeodesic in $D X$. Consequently, Lemma 2.11 implies that $T_{2}$ is $\left(4 \delta_{0}+2 H\left(\delta_{0}, 1,8 \delta_{0}+4 c_{1}\right)\right)$-thin.

Let us prove now that $T$ is also thin. Observe that any point of $T \backslash\left(\left[b^{1}, c\right] \cup\left[c, a^{1}\right]\right)$ has a point in $T_{2}$ at distance least or equal than $2 c_{1}+4 \delta_{0}$, since $X^{\prime}$ is $4 \delta_{0}$-fine. We also have that the points in $\left[b^{1}, c\right]$ and in $\left[c, a^{1}\right]$ are at distance least or equal than $4 \delta_{0}$. Hence, $T$ is $\left(4 c_{1}+12 \delta_{0}+2 H\left(\delta_{0}, 1,8 \delta_{0}+4 c_{1}\right)\right)$-thin.

Theorem 2.9 implies that (2) is equivalent to (5) and that (3) is equivalent to (6).

It is clear that (2) implies (3), with $a_{0}=a$ and $b_{0}=b$. We finish the proof by showing that (3) implies (2). Let us fix $k, l \in J$ and $a \in \eta_{k}, b \in \eta_{l}$. Consider $[a, b],\left[b, b_{0}\right],\left[b_{0}, a_{0}\right],\left[a_{0}, a\right] \subset X$ and $x \in[a, b] \subset X$. Since $X$ is $4 \delta_{0}$-thin and $Q:=\left\{a, b, b_{0}, a_{0}\right\}$ is a geodesic quadrilateral in $X$, given $x \in[a, b]$, we have $d_{X}\left(x,\left[b, b_{0}\right] \cup\left[b_{0}, a_{0}\right] \cup\left[a_{0}, a\right]\right) \leq 8 \delta_{0}$. Consequently, $d_{X}\left(x, \cup_{j \in J} \eta_{j}\right) \leq 8 \delta_{0}+c_{2}$ for every $x \in[a, b]$.

\section{BaCkground in RiEmann SURFACES.}

We denote by $\bar{z}, \Re z$ and $\Im z$, respectively, the conjugate, the real part and the imaginary part of $z$.

Both in this section and in the next one we always work with the Poincaré metric; consequently, curvature is always -1 . In fact, many concepts appearing here (as punctures) only make sense with the Poincaré metric.

Below we collect some definitions concerning Riemann surfaces which will be referred to afterwards.

A non-exceptional Riemann surface $S$ is a Riemann surface whose universal covering space is the unit disk $\mathbb{D}=\{z \in \mathbb{C}:|z|<1\}$, endowed with its Poincaré metric, i.e. the metric obtained by projecting the Poincaré metric of the unit disk $d s=2|d z| /\left(1-|z|^{2}\right)$ or, equivalently, the upper half plane $\mathbb{U}=\{z \in \mathbb{C}: \Im z>0\}$, with the metric $d s=|d z| / \Im z$. With this metric, $S$ is a geodesically complete Riemannian manifold with constant curvature -1 , and therefore $S$ is a geodesic metric space. The only Riemann surfaces which are left out are the sphere, the plane, the punctured plane and the tori. It is easy to study the hyperbolicity of these particular cases.

We have used the word geodesic in the sense of Definition 2.2, that is to say, as a global geodesic or a minimizing geodesic; however, we need now to deal with a special type of local geodesics: simple closed geodesics, which obviously can not be minimizing geodesics. We will continue using the word geodesic with the meaning of Definition 2.2, unless we are dealing with closed geodesics.

A $Y$-piece is a bordered non-exceptional Riemann surface which is conformally equivalent to a sphere without three open disks and whose boundary curves are simple closed geodesics. Given three positive numbers $a, b, c$, there is a unique (up to conformal mapping) $Y$-piece such that their boundary curves have lengths $a, b, c$ (see e.g. [13], p. 109). They are a standard tool for constructing Riemann surfaces. A clear description of these $Y$-pieces and their use is given in [15], Chapter X.3 and [13], Chapter 3.

A generalized $Y$-piece is a non-exceptional Riemann surface (with or without boundary) which is conformally equivalent to a sphere without $n$ open disks and $m$ points, with integers $n, m \geq 0$ such that $n+m=3$, the $n$ boundary curves are simple closed geodesics and the $m$ deleted points are punctures. Notice that a generalized $Y$-piece is topologically the union of a $Y$-piece and $m$ cylinders, with $0 \leq m \leq 3$.

The following spaces are a specially interesting example of Schottky double.

Definition 4.1. A Denjoy domain is a domain $\Omega$ in the Riemann sphere with $\partial \Omega \subset \mathbb{R} \cup\{\infty\}$.

Denjoy domains have a growing interest in Geometric Function Theory (see e.g. [2], [3], [21], [23]).

We only consider Denjoy domains $\Omega$ with at least three boundary points; this fact guarantees that $\Omega$ is a non-exceptional Riemann surface.

If we consider the bordered Riemann surface $X:=\Omega \cap\{z \in \mathbb{C}: \Im z \geq 0\}$ and $\left\{\eta_{j}\right\}_{j \in J}$ the connected components of $X \cap \mathbb{R}$, the Denjoy domain $\Omega$ is the double of $X$. Given a subset $A$ of $\Omega$, we denote by $A^{+}$ the set $A^{+}:=A \cap\{z \in \mathbb{C}: \Im z \geq 0\}$; then, the Denjoy domain $\Omega$ is the double of $\Omega^{+}$, i.e., $\Omega=D \Omega^{+}$. 


\section{Results in Riemann surfaces.}

The following result gives several characterizations of the hyperbolicity of the Denjoy domains. It is an improvement of Theorem 3.2 in the context of this kind of spaces.

In particular, characterization (5) gives that it is sufficient to check the Rips condition just for bigons.

Characterization (3) is also a remarkable improvement of Rips condition in the context of Riemann surfaces, since the amount of geodesics to check is drastically reduced with respect to Rips condition. For example, let us consider an annulus $A_{t}:=\mathbb{C} \backslash([-1,0] \cup[t, \infty))$; it is well known that every annulus is conformally equivalent to $A_{t}$ for some $t>0$. Fix some geodesic $\gamma_{0}$ joining $(-\infty,-1)$ with $(0, t)$. In order to deal with the Rips condition, we need to consider a generic triangle $T$ in $A_{t}$, which is determined by the coordinates of three points, i.e., by six real coordinates; however, (3) allows to deal only with $\gamma_{0}$, which is parameterized by one real coordinate.

Theorem 5.1. Let us consider a Denjoy domain $\Omega$. Then the following conditions are equivalent:

(1) $\Omega$ is $\delta$-hyperbolic.

(2) There exists a constant $c_{1}$ such that for every $k, l \in J$ and $a \in \eta_{k}, b \in \eta_{l}$ we have $d_{\Omega}(z, \mathbb{R}) \leq c_{1}$ for every $z \in[a, b]$.

(3) There exists a constant $c_{2}$ such that for every $k, l \in J$ there exist $a_{0} \in \eta_{k}, b_{0} \in \eta_{l}$ with $d_{\Omega}(z, \mathbb{R}) \leq c_{2}$ for every $z \in\left[a_{0}, b_{0}\right]$.

(4) There exist constants $c_{3}, \alpha, \beta$ such that for every $k, l \in J$ there exist $a_{0} \in \eta_{k}, b_{0} \in \eta_{l}$ with $d_{\Omega}(z, \mathbb{R}) \leq c_{3}$ for every $z$ in some $(\alpha, \beta)$-quasigeodesic joining $a_{0}$ with $b_{0}$.

(5) There exists a constant $c_{4}$ such that every geodesic bigon in $\Omega$ with vertices in $\mathbb{R}$ is $c_{4}$-thin.

Furthermore, the constants in each condition only depend on the constants appearing in any other of the conditions.

Proof. Theorem 5.1 is a consequence of Theorem 3.2 if we consider the bordered Riemann surface $X:=$ $\Omega^{+}=\Omega \cap\{z \in \mathbb{C}: \Im z \geq 0\}$ and $\left\{\eta_{j}\right\}_{j \in J}$ the connected components of $X \cap \mathbb{R}$. We only need to remark two facts:

(a) $X$ is hyperbolic since it is isometric to a geodesically convex subset of the unit disk (in fact, there is just one geodesic in $X$ joining two points in $X$ ). Therefore, $X$ is $\log (1+\sqrt{2})$-thin, as the unit disk (see, e.g. [5], p.130).

(b) If $a, a_{0} \in \eta_{k}, b, b_{0} \in \eta_{l}$, then $\left[a, a_{0}\right]$ and $\left[b, b_{0}\right]$ are subsets of $\mathbb{R}$.

It is obvious that as we focus on more particular kind of surfaces, we can obtain more powerful results. That is the reason because we introduce now a new type of space. However, the following theorems will be extended to a more general context later.

Definition 5.2. A train is a Denjoy domain $\Omega \subset \mathbb{C}$ with $\Omega \cap \mathbb{R}=\cup_{n=0}^{\infty}\left(a_{n}, b_{n}\right)$, such that $-\infty \leq a_{0}$ and $b_{n} \leq a_{n+1}$ for every $n$. A flute surface is a train with $b_{n}=a_{n+1}$ for every $n$.

We say that a curve in a train $\Omega$ is a fundamental geodesic if it is a simple closed geodesic which just intersects $\mathbb{R}$ in $\left(a_{0}, b_{0}\right)$ and $\left(a_{n}, b_{n}\right)$ for some $n>0$; we denote by $\gamma_{n}$ the fundamental geodesic corresponding to $n$ and $2 l_{n}:=L_{\Omega}\left(\gamma_{n}\right)$. A curve in a train $\Omega$ is a second fundamental geodesic if it is a simple closed geodesic which just intersects $\mathbb{R}$ in $\left(a_{n}, b_{n}\right)$ and $\left(a_{n+1}, b_{n+1}\right)$ for some $n \geq 0$; we denote by $\sigma_{n}$ the second fundamental geodesic corresponding to $n$ and $2 r_{n}:=L_{\Omega}\left(\sigma_{n}\right)$. If $b_{n}=a_{n+1}$, we define $\sigma_{n}$ as the puncture at this point and $r_{n}=0$.

$A$ fundamental $Y$-piece in a train $\Omega$ is the generalized $Y$-piece in $\Omega$ bounded by $\gamma_{n}, \gamma_{n+1}, \sigma_{n}$ for some $n>0$; we denote by $Y_{n}$ the fundamental $Y$-piece corresponding to $n$. A fundamental hexagon in a train $\Omega$ is the intersection $H_{n}:=Y_{n}^{+}=Y_{n} \cap\{z \in \mathbb{C}: \Im z \geq 0\}$ for some $n>0$. We denote by $\alpha_{n}$ the length of the opposite side to $\sigma_{n}^{+}$in $H_{n}$.

\section{Remarks.}

1. Observe that $\eta_{n}=\left(a_{n}, b_{n}\right)$ is a closed set in $\Omega$ with $L_{\Omega}\left(\left(a_{n}, b_{n}\right)\right)=\infty$, since $a_{n}, b_{n} \notin \Omega$. 
2. A train is a flute surface if and only if every second fundamental geodesic is a puncture. Flute surfaces are a special case of trains, but they are important by themselves (see, e.g. [7], [8]), since they are the simplest examples of infinite ends; in a flute surface it is possible to give a fairly precise description of the ending geometry (see, e.g. [24]).

Theorem 5.3. Let us consider a train $\Omega$. Then the following conditions are equivalent:

(1) $\Omega$ is $\delta$-hyperbolic.

(2) There exists a constant $c_{1}$ such that $d_{\Omega}(z, \mathbb{R}) \leq c_{1}$ for every $z \in \cup_{n} \gamma_{n}$.

(3) There exist constants $c_{2}, \alpha, \beta$ such that $d_{\Omega}(z, \mathbb{R}) \leq c_{2}$ for every $z \in \cup_{n} g_{n}$, where $g_{n}$ is freely homotopic to $\gamma_{n}, \overline{g_{n}}=g_{n}$ and $g_{n}^{+}$is an $(\alpha, \beta)$-quasigeodesic.

Furthermore, the constants in each condition only depend on the constants appearing in any other of the conditions.

Remark. Recall that $\overline{g_{n}}$ denotes the conjugate of $g_{n}$.

Proof. The equivalence between (2) and (3) is a direct consequence of Theorem 2.9. Theorem 5.1 gives that (1) implies (2).

We prove now that (2) implies (1). By Theorem 5.1, it is enough to prove that there exists a constant $c_{1}^{*}$ such that $d_{\Omega}(z, \mathbb{R}) \leq c_{1}^{*}$ for every $z \in \cup_{n} \gamma_{m n}$, where $\gamma_{m n}$ is the simple closed geodesic which just intersects $\mathbb{R}$ in $\left(a_{m}, b_{m}\right)$ and $\left(a_{n}, b_{n}\right)$ for $0<m<n$. Recall that for each set $A$ in $\Omega$, we denote by $A^{+}$the subset $A^{+}:=A \cap\{z \in \mathbb{C}: \Im z \geq 0\}$. Consider the geodesic hexagon $H_{m n}$ in $X:=\Omega^{+}$, with sides $\gamma_{m n}^{+}, \gamma_{m}^{+}, \gamma_{n}^{+}$, and the three geodesics joining their endpoints which are contained in $\left(a_{0}, b_{0}\right),\left(a_{m}, b_{m}\right)$ and $\left(a_{n}, b_{n}\right)$. Since $X$ is isometric to a geodesically convex subset of the unit disk, it is $\log (1+\sqrt{2})$-thin, as the unit disk. Hence, $H_{m n}$ is $4 \log (1+\sqrt{2})$-thin, and given any $z \in \gamma_{m n}^{+}$, there exists $z_{0} \in \gamma_{m}^{+} \cup \gamma_{n}^{+} \cup \mathbb{R}$ with $d_{\Omega}\left(z, z_{0}\right) \leq 4 \log (1+\sqrt{2})$. Therefore, $d_{\Omega}(z, \mathbb{R}) \leq c_{1}+4 \log (1+\sqrt{2})$. By symmetry, $d_{\Omega}(z, \mathbb{R}) \leq c_{1}+4 \log (1+\sqrt{2})$ holds for every $z \in \gamma_{m n}$.

This proof gives directly the following.

Corollary 5.4. Let us consider a Denjoy domain $\Omega$ such that $\cup_{n=0}^{\infty}\left(a_{n}, b_{n}\right) \subseteq \Omega$, with $-\infty \leq a_{0}, b_{n} \leq a_{n+1}$ and $a_{n}, b_{n} \in \partial \Omega$ for every $n$. We denote by $\gamma_{m n}$ the simple closed geodesic joining $\left(a_{m}, b_{m}\right)$ and $\left(a_{n}, b_{n}\right)$, and $\gamma_{n}:=\gamma_{0 n}$. If $d_{\Omega}(z, \mathbb{R}) \leq c_{1}$ for every $z \in \cup_{n} \gamma_{n}$, then $d_{\Omega}(z, \mathbb{R}) \leq c_{1}+4 \log (1+\sqrt{2})$ holds for every $z \in \cup_{m \neq n} \gamma_{m n}$.

Next, some lemmas which will allow us to study the hyperbolicity of trains in terms of the lengths of their fundamental geodesics.

Lemma 5.5. Let us consider a train $\Omega$.

(1) We have for every $n$,

$$
(\operatorname{Arcsinh} 2)\left(e^{-l_{n}}+e^{-l_{n+1}}\right) \leq \alpha_{n}
$$

(2) If $r_{n} \leq c_{1}+\left|l_{n}-l_{n+1}\right|$ and $l_{n}, l_{n+1} \geq l_{0}$ for some fixed $n$, then there exists a constant $c_{2}$, which only depends on $c_{1}$ and $l_{0}$, such that

$$
\alpha_{n} \leq c_{2}\left(e^{-l_{n}}+e^{-l_{n+1}}\right) .
$$

Proof. Standard hyperbolic trigonometry (see e.g. [9], p. 161) gives

$$
\cosh \alpha_{n}=\frac{\cosh r_{n}+\cosh l_{n} \cosh l_{n+1}}{\sinh l_{n} \sinh l_{n+1}} .
$$


Since $\operatorname{coth} t \geq 1+2 e^{-2 t}$ if $t \geq 0$, we obtain

$$
\begin{aligned}
\cosh \alpha_{n} & \geq 4 e^{-l_{n}-l_{n+1}}+\left(1+2 e^{-2 l_{n}}\right)\left(1+2 e^{-2 l_{n+1}}\right), \\
\cosh \alpha_{n} & \geq 4 e^{-l_{n}-l_{n+1}}+1+2 e^{-2 l_{n}}+2 e^{-2 l_{n+1}}, \\
\sinh ^{2} \frac{\alpha_{n}}{2} & =\frac{\cosh \alpha_{n}-1}{2} \geq e^{-2 l_{n}}+e^{-2 l_{n+1}}+2 e^{-l_{n}-l_{n+1}}, \\
\sinh \frac{\alpha_{n}}{2} & \geq e^{-l_{n}}+e^{-l_{n+1}}, \\
\alpha_{n} & \geq 2 \operatorname{Arcsinh}\left(e^{-l_{n}}+e^{-l_{n+1}}\right) .
\end{aligned}
$$

Since the function $t^{-1} \operatorname{Arcsinh} t$ is decreasing in $[0,2]$, we have

$$
2 \operatorname{Arcsinh} t \geq t \operatorname{Arcsinh} 2 \quad \forall t \in[0,2] \quad \text { and } \quad \alpha_{n} \geq(\operatorname{Arcsinh} 2)\left(e^{-l_{n}}+e^{-l_{n+1}}\right) .
$$

This finishes the proof of (1).

In order to prove $(2)$, we remark that if $x \geq l_{0}$, then $e^{-2 l_{0}} e^{2 x} \geq 1$ and $e^{2 x}-1 \geq\left(1-e^{-2 l_{0}}\right) e^{2 x}$. Therefore, if we define $c_{3}^{-1}:=\left(1-e^{-2 l_{0}}\right) / 2$, we have

$$
e^{2 x}-1 \geq 2 c_{3}^{-1} e^{2 x}, \quad \sinh x \geq c_{3}^{-1} e^{x}, \quad \operatorname{coth} x=1+\frac{2}{e^{2 x}-1} \leq 1+c_{3} e^{-2 x}, \quad \text { for every } x \geq l_{0} .
$$

Hence, we obtain

$$
\cosh \alpha_{n}=\frac{\cosh r_{n}+\cosh l_{n} \cosh l_{n+1}}{\sinh l_{n} \sinh l_{n+1}} \leq c_{3}^{2} e^{r_{n}-l_{n}-l_{n+1}}+\left(1+c_{3} e^{-2 l_{n}}\right)\left(1+c_{3} e^{-2 l_{n+1}}\right) .
$$

The inequality $r_{n}-l_{n}-l_{n+1} \leq-2 \min \left\{l_{n}, l_{n+1}\right\}+c_{1}$ (which is equivalent to $r_{n} \leq c_{1}+\left|l_{n}-l_{n+1}\right|$ ) gives

$$
c_{3}^{2} e^{r_{n}-l_{n}-l_{n+1}} \leq c_{3}^{2} e^{c_{1}-2 \min \left\{l_{n}, l_{n+1}\right\}} \leq c_{3}^{2} e^{c_{1}}\left(e^{-2 l_{n}}+e^{-2 l_{n+1}}\right) .
$$

Then

$$
\begin{aligned}
\cosh \alpha_{n} & \leq c_{3}^{2} e^{c_{1}}\left(e^{-2 l_{n}}+e^{-2 l_{n+1}}\right)+1+c_{3} e^{-2 l_{n}}+c_{3} e^{-2 l_{n+1}}+c_{3}^{2} e^{-2 l_{n}-2 l_{n+1}}, \\
2 \sinh ^{2} \frac{\alpha_{n}}{2} & =\cosh \alpha_{n}-1 \leq\left(c_{3}^{2} e^{c_{1}}+c_{3}+c_{3}^{2}\right)\left(e^{-2 l_{n}}+e^{-2 l_{n+1}}\right), \\
\frac{\alpha_{n}}{2} & \leq \sinh \frac{\alpha_{n}}{2} \leq \frac{c_{2}}{2}\left(e^{-l_{n}}+e^{-l_{n+1}}\right),
\end{aligned}
$$

and we obtain $\alpha_{n} \leq c_{2}\left(e^{-l_{n}}+e^{-l_{n+1}}\right)$.

Definition 5.6. Given a train $\Omega$ and a point $z \in \Omega$, we define the height of $z$ as $h(z):=d_{\Omega}\left(z,\left(a_{0}, b_{0}\right)\right)$. We define $z_{0}$ as the point in $\left(a_{0}, b_{0}\right)$ with $h(z)=d_{\Omega}\left(z,\left(a_{0}, b_{0}\right)\right)=d_{\Omega}\left(z, z_{0}\right)$. We denote by $p(z)$ a real number with $d_{\Omega}(z, p(z))=d_{\Omega}(z, \mathbb{R})$. (It is possible that there exist several real numbers with this property; in this case $p(z)$ denotes any choice.)

Lemma 5.7. Let us consider a train $\Omega$. We have $d_{\Omega}(z, w) \geq|h(z)-h(w)|$ for every $z, w \in \Omega$. Furthermore, if $\Omega$ is $\delta$-hyperbolic, then there exists a constant $c$, which only depends on $\delta$, such that $|h(z)-h(p(z))| \leq c$ for every $z \in \cup_{n} \gamma_{n}$.

Proof. Fix $z \in \Omega$. It is enough to show that $d_{\Omega}(z, w) \geq|h(z)-h(w)|$; the second part of the lemma is a consequence of this fact (with $w=p(z)$ ) and Theorem 5.3. Let us consider the geodesic quadrilateral $\left\{z, w, w_{0}, z_{0}\right\}$. Standard hyperbolic trigonometry (see e.g. [18], p.88) gives

$$
\begin{aligned}
\cosh d_{\Omega}(z, w) & =\cosh d_{\Omega}\left(z_{0}, w_{0}\right) \cosh h(z) \cosh h(w)-\sinh h(z) \sinh h(w) \\
& \geq \cosh h(z) \cosh h(w)-\sinh h(z) \sinh h(w)=\cosh (h(z)-h(w)),
\end{aligned}
$$

and consequently, $d_{\Omega}(z, w) \geq|h(z)-h(w)|$.

Lemma 5.8. Let us consider a train $\Omega$. If $l_{0} \leq l_{n}<l_{n+1}$ and $r_{n} \leq c_{1}$ for some fixed $n$, then $d_{\Omega}(z, \mathbb{R}) \leq$ $d_{\Omega}\left(z,\left(a_{n}, b_{n}\right)\right) \leq c_{2}$ for every $z \in \gamma_{n+1}$ with $h(z) \in\left[l_{n}, l_{n+1}\right]$, where $c_{2}$ only depends on $c_{1}$ and $l_{0}$. We also have $d_{\Omega}\left(z, \mathbb{R} \cup \gamma_{n}\right) \leq c_{2}$ for every $z \in \gamma_{n+1}$. 
Proof. By Lemma 5.5 there exists a constant $c_{3}$, which only depends on $c_{1}$ and $l_{0}$, such that $\alpha_{n} \leq c_{3} / 2\left(e^{-l_{n}}+\right.$ $\left.e^{-l_{n+1}}\right)$. We have $\alpha_{n} \leq c_{3} e^{-l_{n}}$ and $\sinh \alpha_{n} \leq e^{-l_{n}} \sinh c_{3}$, since $\sinh a t \leq t \sinh a$ for every $t \in[0,1]$.

Fix $z \in \gamma_{n+1}$ with $h(z) \in\left[l_{n}, l_{n+1}\right]$. By symmetry, without loss of generality we can assume that $z \in \gamma_{n+1}^{+}$.

Let us define $u^{n}:=\left(a_{n}, b_{n}\right) \cap \gamma_{n}, u^{n+1}:=\left(a_{n+1}, b_{n+1}\right) \cap \gamma_{n+1}$ and $v^{n}$ as the point in $\gamma_{n+1}^{+}$with $d_{\Omega}\left(u^{n}, \gamma_{n+1}\right)=d_{\Omega}\left(u^{n}, v^{n}\right)$. By convexity (see e.g. [10], section 4, or [17], p.2), it is clear that $d_{\Omega}\left(z,\left(a_{n}, b_{n}\right)\right) \leq$ $\max \left\{d_{\Omega}\left(u^{n}, \gamma_{n+1}\right), d_{\Omega}\left(u^{n+1},\left(a_{n}, b_{n}\right)\right)\right\}$. It is also clear that $d_{\Omega}\left(z, \mathbb{R} \cup \gamma_{n}\right) \leq \max \left\{d_{\Omega}\left(u^{n}, \gamma_{n+1}\right), d_{\Omega}\left(u^{n+1},\left(a_{n}, b_{n}\right)\right)\right\}$, if $h(z) \leq l_{n}$.

Let us consider the geodesic right-angled quadrilateral $\left\{u^{n}, u_{0}^{n}, v_{0}^{n}, v^{n}\right\}$. Standard hyperbolic trigonometry (see e.g. [18], p.88) gives $\sinh d_{\Omega}\left(u^{n}, \gamma_{n+1}\right)=\sinh \alpha_{n} \cosh l_{n}<e^{-l_{n}} \sinh c_{3} e^{l_{n}}=\sinh c_{3}$, and consequently, $d_{\Omega}\left(u^{n}, \gamma_{n+1}\right)<c_{3}$.

The shortest geodesic in $H_{n}$ joining $\left(a_{n}, b_{n}\right)$ with $\gamma_{n+1}^{+}$separates $H_{n}$ into two right-angled pentagons: $P_{n}$ (which contains $\gamma_{n}^{+}$) and $Q_{n}$ (which contains $\sigma_{n}^{+}$). We denote by $w^{n+1}$ the intersection of this geodesic with $\gamma_{n+1}^{+}$. Considering $P_{n}$, standard hyperbolic trigonometry (see e.g. [18], p.87) gives $\sinh l_{n} \sinh \alpha_{n}=$ $\cosh d_{\Omega}\left(\gamma_{n+1}^{+},\left(a_{n}, b_{n}\right)\right)$, and then

$$
\sinh d_{\Omega}\left(\gamma_{n+1}^{+},\left(a_{n}, b_{n}\right)\right)<\cosh d_{\Omega}\left(\gamma_{n+1}^{+},\left(a_{n}, b_{n}\right)\right)=\sinh l_{n} \sinh \alpha_{n} \leq e^{l_{n}} e^{-l_{n}} \sinh c_{3}=\sinh c_{3} .
$$

Hence, $d_{\Omega}\left(\gamma_{n+1}^{+},\left(a_{n}, b_{n}\right)\right)<c_{3}$. Considering $Q_{n}$, standard hyperbolic trigonometry gives $\sinh d_{\Omega}\left(\gamma_{n+1}^{+},\left(a_{n}, b_{n}\right)\right) \sinh d_{\Omega}\left(u^{n+1}, w^{n+1}\right)=\cosh r_{n}$, and then

$$
\sinh d_{\Omega}\left(u^{n+1}, w^{n+1}\right)=\frac{\cosh r_{n}}{\sinh d_{\Omega}\left(\gamma_{n+1}^{+},\left(a_{n}, b_{n}\right)\right)} \leq \frac{\cosh c_{1}}{\sinh d_{\Omega}\left(\gamma_{n+1}^{+},\left(a_{n}, b_{n}\right)\right)} .
$$

The shortest geodesic in $Q_{n}$ joining $u^{n+1}$ with $\left(a_{n}, b_{n}\right)$ separates $Q_{n}$ into two right-angled quadrilaterals. Considering the right-angled quadrilateral which contains $\gamma_{n+1} \cap Q_{n}$, standard hyperbolic trigonometry gives

$$
\begin{aligned}
\sinh d_{\Omega}\left(u^{n+1},\left(a_{n}, b_{n}\right)\right) & =\sinh d_{\Omega}\left(\gamma_{n+1}^{+},\left(a_{n}, b_{n}\right)\right) \cosh d_{\Omega}\left(u^{n+1}, w^{n+1}\right) \\
& \leq \sinh d_{\Omega}\left(\gamma_{n+1}^{+},\left(a_{n}, b_{n}\right)\right) \sqrt{\frac{\cosh ^{2} c_{1}}{\sinh ^{2} d_{\Omega}\left(\gamma_{n+1}^{+},\left(a_{n}, b_{n}\right)\right)}+1} \\
& \leq \sqrt{\cosh ^{2} c_{1}+\sinh ^{2} d_{\Omega}\left(\gamma_{n+1}^{+},\left(a_{n}, b_{n}\right)\right)} \leq \sqrt{\cosh ^{2} c_{1}+\sinh ^{2} c_{3}},
\end{aligned}
$$

and consequently, $d_{\Omega}\left(u^{n+1},\left(a_{n}, b_{n}\right)\right) \leq c_{4}$. If $c_{2}:=\max \left\{c_{3}, c_{4}\right\}$, then $d_{\Omega}(z, \mathbb{R}) \leq d_{\Omega}\left(z,\left(a_{n}, b_{n}\right)\right) \leq c_{2}$, if $h(z) \in\left[l_{n}, l_{n+1}\right]$, and $d_{\Omega}\left(z, \mathbb{R} \cup \gamma_{n}\right) \leq c_{2}$, if $h(z) \leq l_{n}$.

Lemma 5.9. Let us consider a train $\Omega$. If $r_{n_{0}} \leq c_{1}$, then $d_{\Omega}\left(z, \mathbb{R} \cup \gamma_{n_{0}}\right) \leq 4 \log (1+\sqrt{2})+c_{1} / 2$ for every $z \in \gamma_{n_{0}+1}$.

Proof. Let us consider $z \in \gamma_{n_{0}+1}$. Without loss of generality we can assume that $z \in \gamma_{n_{0}+1}^{+} \subset H_{n_{0}}=Y_{n_{0}}^{+}$. Since $H_{n_{0}}$ is a (simply connected) right-angled hexagon, it is isometric to a hexagon in the unit disk. Every hexagon in the unit disk is $4 \log (1+\sqrt{2})$-thin, since the unit disk is $\log (1+\sqrt{2})$-thin (see, e.g. [5], p.130). Let us denote by $w$ a point in $\partial H_{n_{0}} \backslash \gamma_{n_{0}+1}^{+}$with $d_{\Omega}(z, w) \leq 4 \log (1+\sqrt{2})$. If $w \in \mathbb{R} \cup \gamma_{n_{0}}$, the conclusion of the lemma holds. If $w \in \sigma_{n_{0}}^{+}$, then $d_{\Omega}(w, \mathbb{R}) \leq c_{1} / 2$ and $d_{\Omega}\left(z, \mathbb{R} \cup \gamma_{n_{0}}\right) \leq 4 \log (1+\sqrt{2})+c_{1} / 2$.

The following lemma gathers the main ideas and computations which will be applied in the theorems below.

Lemma 5.10. Let us consider a train $\Omega$.

(a) If there exists a (finite or infinite) subset $\left\{n_{k}\right\}_{k} \subset \mathbb{N}$ with $r_{n} \leq c_{1}+\left|l_{n}-l_{n+1}\right|$ for every $n \in$ $\left[n_{1}, \sup _{k} n_{k}\right)$, and $l_{n} \geq l_{0}>0$ for every $n \in\left[n_{1}, \sup _{k} n_{k}\right], l_{n_{1}} \leq l^{0}, r_{n_{k}} \leq c_{1}, l_{n_{k}+1}+c_{1} \geq l_{n_{k+1}}$ for every $k$, and 


$$
\sum_{n=n_{m}+1}^{n_{k}} e^{-l_{n}} \leq c_{2} e^{-l_{n_{m}+1}}, \quad \text { for every } m<k
$$

then $d_{\Omega}(z, \mathbb{R}) \leq c$ for every $z \in \cup_{k} \gamma_{n_{k}}$ with $h(z)>l_{n_{1}}$, where $c$ is a constant which only depends on $c_{1}$, $c_{2}$ and $l_{0}$. Consequently, $d_{\Omega}(z, \mathbb{R}) \leq \max \left\{c, l^{0}\right\}$ for every $z \in \cup_{k} \gamma_{n_{k}}$. We also have $d_{\Omega}\left(z, \mathbb{R} \cup \gamma_{n_{m}}\right) \leq c$ for every $m$ and every $z \in \cup_{k \geq m} \gamma_{n_{k}}$.

(b) If $\lim _{n \rightarrow \infty} l_{n}=\infty,\left\{n_{k}\right\}_{k}$ is a subsequence with $l_{n}+c_{3} \geq l_{n_{k}}$ for every $k$ and every $n \geq n_{k}$, and such that the condition

$$
\sum_{n=n_{k}}^{\infty} e^{-l_{n}} \leq c_{2} e^{-l_{n_{k}}}, \quad \text { for every } k,
$$

does not hold for this $\left\{n_{k}\right\}_{k}$, then $\Omega$ is not hyperbolic.

Proof. We prove first $(a)$. Fix $z \in \gamma_{n_{k}}$ for some $k$, with $h(z)>l_{n_{1}}$. By symmetry, without loss of generality we can assume that $z \in \gamma_{n_{k}}^{+}$.

By Lemma 5.5, there exists a constant $c_{4}$, which only depends on $c_{1}$ and $l_{0}$, such that $\alpha_{n} \leq c_{4} / 2\left(e^{-l_{n}}+\right.$ $\left.e^{-l_{n+1}}\right)$ for any $n \in\left[n_{1}, \sup _{k} n_{k}\right)$.

Since $h(z)>l_{n_{1}}$, we can choose $1 \leq m<k$ verifying both $l_{n_{m}}<l_{n_{m+1}}$ and $h(z) \in\left[l_{n_{m}}, l_{n_{m+1}}\right]$.

If $h(z) \geq l_{n_{m}+1}$, consider the point $z^{*} \in\left(a_{n_{m}+1}, b_{n_{m}+1}\right) \cap H_{n_{m}+1}$ with $h(z)=h\left(z^{*}\right)$. If $h(z)<l_{n_{m}+1}$, we consider the point $z^{*} \in \gamma_{n_{m}+1}^{+}$with $h(z)=h\left(z^{*}\right)$.

In both cases, we take the geodesic quadrilateral $\left\{z, z^{*}, z_{0}^{*}, z_{0}\right\}$. Standard hyperbolic trigonometry gives $\sinh \frac{1}{2} d_{\Omega}\left(z, z^{*}\right)=\sinh \frac{1}{2} d_{\Omega}\left(z_{0}, z_{0}^{*}\right) \cosh h(z)$. Observe that

$$
d_{\Omega}\left(z_{0}, z_{0}^{*}\right) \leq \sum_{n=n_{m}+1}^{n_{k}-1} \alpha_{n} \leq \sum_{n=n_{m}+1}^{n_{k}-1} \frac{c_{4}}{2}\left(e^{-l_{n}}+e^{-l_{n+1}}\right) \leq \sum_{n=n_{m}+1}^{n_{k}} c_{4} e^{-l_{n}} \leq c_{2} c_{4} e^{-l_{n_{m}+1}},
$$

and therefore $d_{\Omega}\left(z_{0}, z_{0}^{*}\right)$ is bounded by $c_{2} c_{4}$. Then, $\sinh \frac{1}{2} d_{\Omega}\left(z_{0}, z_{0}^{*}\right) \leq c_{5} e^{-l_{n_{m}+1}}$ and $\sinh \frac{1}{2} d_{\Omega}\left(z, z^{*}\right) \leq$ $c_{5} e^{-l_{n_{m}+1}} e^{h(z)} \leq c_{5} e^{c_{1}-l_{n_{m+1}}} e^{h(z)} \leq c_{5} e^{c_{1}}$. If $h(z) \geq l_{n_{m}+1}$, then $d_{\Omega}(z, \mathbb{R}) \leq 2 \operatorname{Arcsinh}\left(c_{5} e^{c_{1}}\right)$.

If $h(z)<l_{n_{m}+1}$, then $z^{*} \notin \mathbb{R}$, but we have $h(z) \in\left[l_{n_{m}}, l_{n_{m}+1}\right]$. Hence, Lemma 5.8 gives $d_{\Omega}\left(z^{*}, \mathbb{R}\right) \leq$ $d_{\Omega}\left(z^{*},\left(a_{n_{m}}, b_{n_{m}}\right)\right) \leq c_{6}$, where $c_{6}$ only depends on $c_{1}$ and $l_{0}$.

Consequently, $d_{\Omega}(z, \mathbb{R}) \leq d_{\Omega}\left(z, z^{*}\right)+d_{\Omega}\left(z^{*}, \mathbb{R}\right) \leq 2 \operatorname{Arcsinh}\left(c_{5} e^{c_{1}}\right)+c_{6}=: c$, for every $z \in \cup_{k} \gamma_{n_{k}}$ with $h(z)>l_{n_{1}}$.

The same computations finish the proof of part (a) (recall that Lemma 5.8 also covers the case $h(z) \leq l_{n_{1}}$ ).

We prove now $(b)$. By Lemma 5.7, without loss of generality we can assume that there exists a constant $c_{7}$ such that $|h(z)-h(p(z))| \leq c_{7}$ for every $z \in \cup_{n} \gamma_{n}$. Since (5.2) does not hold, given any $M>e^{2\left(c_{3}+c_{7}\right)}$, there exist $m<k$ such that

$$
\sum_{n=n_{m}}^{n_{k}} e^{-l_{n}} \geq M e^{-l_{n_{m}}}
$$

Since $\lim _{n \rightarrow \infty} l_{n}=\infty$, without loss of generality we can take $m$ large enough so that $l_{n_{m}} \geq \log M$. Consider $z \in \gamma_{n_{k}}^{+}$with $h(z)=l_{n_{m}}-\frac{1}{2} \log M<l_{n_{m}}-c_{3}-c_{7}$; hence, $h(p(z))<l_{n_{m}}-c_{3} \leq l_{n}$ for every $n \geq n_{m}$, and $p(z) \in \cup_{n=0}^{n_{m}-1}\left(a_{n}, b_{n}\right)$. We also have $h(z)=d_{\Omega}\left(z,\left(a_{0}, b_{0}\right)\right)=l_{n_{m}}-\frac{1}{2} \log M \geq \frac{1}{2} \log M>c_{7}$, and then $p(z) \notin\left(a_{0}, b_{0}\right)$.

Since $p(z) \in \cup_{n=1}^{n_{m}-1}\left(a_{n}, b_{n}\right)$, let us consider the geodesic quadrilateral $\left\{z, p(z), p(z)_{0}, z_{0}\right\}$. Standard hyperbolic trigonometry gives

$$
\begin{aligned}
\cosh d_{\Omega}(z, p(z)) & =\cosh d_{\Omega}\left(z_{0}, p(z)_{0}\right) \cosh h(z) \cosh h(p(z))-\sinh h(z) \sinh h(p(z)) \\
& \geq\left(\cosh d_{\Omega}\left(z_{0}, p(z)_{0}\right)-1\right) \cosh h(z) \cosh h(p(z)) \\
& \geq \frac{1}{8} d_{\Omega}\left(z_{0}, p(z)_{0}\right)^{2} e^{h(z)} e^{h(p(z))} .
\end{aligned}
$$


Observe that, by Lemma 5.5,

$$
d_{\Omega}\left(z_{0}, p(z)_{0}\right) \geq d_{\Omega}\left(z_{0}, \gamma_{n_{m}}\right) \geq \sum_{n=n_{m}}^{n_{k}-1} \alpha_{n} \geq \sum_{n=n_{m}}^{n_{k}-1}\left(e^{-l_{n}}+e^{-l_{n+1}}\right)>\sum_{n=n_{m}}^{n_{k}} e^{-l_{n}} \geq M e^{-l_{n_{m}}} .
$$

Consequently,

$$
\cosh d_{\Omega}(z, \mathbb{R})=\cosh d_{\Omega}(z, p(z)) \geq \frac{1}{8} M^{2} e^{-2 l_{n_{m}}} e^{l_{n_{m}}-\frac{1}{2} \log M} e^{l_{n_{m}}-\frac{1}{2} \log M-c_{7}}=\frac{1}{8} M e^{-c_{7}} .
$$

Since $M$ can be arbitrarily large, Theorem 5.3 gives that $\Omega$ is not hyperbolic.

Corollary 5.11. Let us consider a train $\Omega$ with $r_{n} \leq c_{1}$ for every $M \leq n<N, l_{M} \leq l^{0}$ and

$$
\sum_{k=n}^{N} e^{-l_{k}} \leq c_{2} e^{-l_{n}}, \quad \text { for every } M<n \leq N .
$$

Then $d_{\Omega}\left(z, \mathbb{R} \cup \gamma_{M}\right) \leq c$ for every $z \in \cup_{n=M}^{N} \gamma_{n}$, where $c$ is a constant which only depends on $c_{1}$ and $c_{2}$. Consequently, $d_{\Omega}(z, \mathbb{R}) \leq \max \left\{c, l^{0}\right\}$ for every $z \in \cup_{n=M}^{N} \gamma_{n}$.

Proof. If $l_{n}<1$ for some $M \leq n \leq N$, then $d_{\Omega}\left(z, \mathbb{R} \cup \gamma_{M}\right) \leq d_{\Omega}(z, \mathbb{R}) \leq 1 / 2$ for every $z \in \gamma_{n}$.

Let us consider $M_{0} \leq N_{0}$ with the following properties:

(i) $l_{n} \geq 1$ for every $M_{0} \leq n \leq N_{0}$,

(ii) $l_{M_{0}-1}<1$ or $M_{0}=M$

(iii) $l_{N_{0}+1}<1$ or $N_{0}=N$.

Part $(a)$ of Lemma 5.10, with $\left\{n_{k}\right\}_{k}=\left\{M_{0}, M_{0}+1, \ldots, N_{0}\right\}$ (observe that in this case $n_{k+1}=n_{k}+1$ ), gives that $d_{\Omega}\left(z, \mathbb{R} \cup \gamma_{M_{0}}\right) \leq c_{3}$ for every $z \in \cup_{n=M_{0}}^{N_{0}} \gamma_{n}$, where $c_{3}$ is a constant which only depends on $c_{1}$ and $c_{2}$.

If $M_{0}=M$, then $d_{\Omega}\left(z, \mathbb{R} \cup \gamma_{M}\right) \leq c_{3}$ for every $z \in \cup_{n=M_{0}}^{N_{0}} \gamma_{n}$.

If $M_{0}>M$, then $l_{M_{0}-1}<1$. Lemma 5.9 gives that $d_{\Omega}\left(z, \mathbb{R} \cup \gamma_{M_{0}-1}\right) \leq 4 \log (1+\sqrt{2})+c_{1} / 2$ for every $z \in \gamma_{M_{0}}$. Hence, $d_{\Omega}\left(z, \mathbb{R} \cup \gamma_{M}\right) \leq d_{\Omega}(z, \mathbb{R}) \leq c:=c_{3}+4 \log (1+\sqrt{2})+c_{1} / 2+1 / 2$ for every $z \in \cup_{n=M_{0}}^{N_{0}} \gamma_{n}$.

Since every $M \leq n \leq N$ holds either $l_{n}<1$ or $M_{0} \leq n \leq N_{0}$, for some $M_{0} \leq N_{0}$ verifying $(i)$, (ii) and (iii), then $d_{\Omega}\left(z, \mathbb{R} \cup \gamma_{M}\right) \leq c$ for every $z \in \cup_{n=M}^{N} \gamma_{n}$.

Now, we provide the results that study hyperbolicity in terms of $\left\{l_{n}\right\}_{n}$ and $\left\{r_{n}\right\}_{n}$. We deal separately the cases when $\lim _{n \rightarrow \infty} l_{n}=\infty,\left\{l_{n}\right\}_{n}$ is bounded, or none of these. Firs of all, we consider when $\lim _{n \rightarrow \infty} l_{n}=\infty$.

Theorem 5.12. Let us consider a train $\Omega$ with $\lim _{n \rightarrow \infty} l_{n}=\infty$.

(a) If $l_{1} \leq l^{0}, r_{n} \leq c_{1}$ for every $n$ and

$$
\sum_{k=n}^{\infty} e^{-l_{k}} \leq c_{2} e^{-l_{n}}, \quad \text { for every } n>1
$$

then $\Omega$ is $\delta$-hyperbolic, where $\delta$ is a constant which only depends on $c_{1}, c_{2}$ and $l^{0}$.

(b) If $l_{n}+c_{3} \geq l_{m}$ for every $n \geq m$ and $\Omega$ is hyperbolic, then (5.4) holds.

\section{Remarks.}

1. Condition (5.4) is equivalent to

$$
\limsup _{n \rightarrow \infty} e^{l_{n}} \sum_{k=n}^{\infty} e^{-l_{k}}<\infty
$$

2. Examples of sequences verifying this property are $l_{n}=a^{n^{b}}(a>1, b>0)$, and $l_{n}=n^{a}(a \geq 1)$. Examples of sequences that do not verify this property are $l_{n}=n^{a}(a<1)$, and $l_{n}=a \log n(a>0)$.

3. Condition $l_{n}+c_{3} \geq l_{m}$ for every $n \geq m$ holds, for example, if $\left\{l_{n}\right\}$ is a non-decreasing sequence. 
Proof. By Corollary 5.11, for any fixed $N$, we have $d_{\Omega}(z, \mathbb{R}) \leq c_{3}$ for every $z \in \cup_{n=1}^{N} \gamma_{n}$, where $c_{3}$ is a constant which only depends on $c_{1}, c_{2}$ and $l^{0}$. Since $c_{3}$ does not depend on $N$, we obtain $d_{\Omega}(z, \mathbb{R}) \leq c_{3}$ for every $z \in \cup_{n=1}^{\infty} \gamma_{n}$. Then $\Omega$ is $\delta$-hyperbolic by Theorem 5.3 , where $\delta$ is a constant which only depends on $c_{1}$, $c_{2}$ and $l^{0}$.

Assume now that (5.4) does not hold. If we take $\left\{n_{k}\right\}_{k}=\mathbb{N}$, part $(b)$ of Lemma 5.10 gives that $\Omega$ is not hyperbolic.

Corollary 5.13. Let us consider a train $\Omega$, with $\lim _{n \rightarrow \infty} l_{n}=\infty, l_{n}+c_{1} \geq l_{m}$ for every $n \geq m$, and $\sum_{n=1}^{\infty} e^{-l_{n}}=\infty$. Then $\Omega$ is not hyperbolic.

We obtain directly the following characterization.

Theorem 5.14. Let us consider a train $\Omega$ with $\lim _{n \rightarrow \infty} l_{n}=\infty, r_{n} \leq c_{1}$ for every $n$, and $l_{n}+c_{1} \geq l_{m}$ for every $n \geq m$. Then $\Omega$ is hyperbolic if and only if (5.4) holds.

The following theorem shows that the hypothesis $r_{n} \leq c_{1}$ is not very restrictive if $\lim _{n \rightarrow \infty} l_{n}=\infty$. (This is not the case if we have $l_{n} \leq c$; see Theorem 5.25.) We need two lemmas.

Lemma 5.15. Let us consider a train $\Omega$. Assume that $l_{n_{0}} \leq l_{n}+c$ for every $n \geq n_{0}$. Then $d_{\Omega}(z, \mathbb{R}) \leq$ $d_{\Omega}\left(z, \cup_{n=0}^{n_{0}}\left(a_{n}, b_{n}\right)\right)<d_{\Omega}(z, \mathbb{R})+c$ for every $z \in \cup_{n=1}^{n_{0}} \gamma_{n}$.

Proof. The first inequality is trivial. Let us consider $z \in \gamma_{n}$, with $1 \leq n \leq n_{0}$ and such that $d_{\Omega}(z, \mathbb{R})<$ $d_{\Omega}\left(z, \cup_{n=0}^{n_{0}}\left(a_{n}, b_{n}\right)\right)$. Without loss of generality we can assume that $z \in \gamma_{n}^{+}$. We have that $p(z) \in\left(a_{n_{1}}, b_{n_{1}}\right)$, with $n_{1}>n_{0}$; therefore $h(p(z)) \geq l_{n_{1}} \geq l_{n_{0}}-c$. Let us observe that $h(p(z)) \leq l_{n_{0}}$, since if $h(p(z))>l_{n_{0}}$, then $d_{\Omega}(z, \mathbb{R})<d_{\Omega}\left(z,\left(a_{n_{0}}, b_{n_{0}}\right)\right)<d_{\Omega}(z, p(z))=d_{\Omega}(z, \mathbb{R})$, which is a contradiction. Consider the point $z^{\prime} \in \gamma_{n_{0}}^{+}$ with $h\left(z^{\prime}\right)=h(p(z))$. It is clear that $d_{\Omega}\left(z, z^{\prime}\right)<d_{\Omega}(z, p(z))=d_{\Omega}(z, \mathbb{R})$. We also have $d_{\Omega}\left(z^{\prime},\left(a_{n_{0}}, b_{n_{0}}\right)\right)=$ $l_{n_{0}}-h\left(z^{\prime}\right)=l_{n_{0}}-h(p(z)) \leq c$. Consequently,

$$
d_{\Omega}\left(z, \cup_{n=0}^{n_{0}}\left(a_{n}, b_{n}\right)\right) \leq d_{\Omega}\left(z,\left(a_{n_{0}}, b_{n_{0}}\right)\right) \leq d_{\Omega}\left(z, z^{\prime}\right)+d_{\Omega}\left(z^{\prime},\left(a_{n_{0}}, b_{n_{0}}\right)\right)<d_{\Omega}(z, \mathbb{R})+c .
$$

Lemma 5.16. Let us consider a train $\Omega$ and some fixed $n$. We take $z_{n} \in \gamma_{n+1}^{+}$with $h\left(z_{n}\right)=l_{n+1}-s_{n}$, where $s_{n}:=\log \left(\min \left\{l_{n+1}, r_{n}\right\}\right)$. Then

$$
\begin{gathered}
d_{\Omega}\left(z_{n},\left(a_{0}, b_{0}\right)\right) \geq l_{n+1}-\log l_{n+1}, \quad d_{\Omega}\left(z_{n},\left(a_{n}, b_{n}\right)\right) \geq r_{n}-\log r_{n}, \\
d_{\Omega}\left(z_{n}, \gamma_{n}\right) \geq \operatorname{Arcsinh} e^{\frac{1}{2}\left(r_{n}+l_{n+1}-l_{n}-2 \log r_{n}\right) .}
\end{gathered}
$$

Proof. It is direct that $d_{\Omega}\left(z_{n},\left(a_{0}, b_{0}\right)\right)=h\left(z_{n}\right)=l_{n+1}-s_{n} \geq l_{n+1}-\log l_{n+1}$. We also have that $r_{n}=$ $d_{\Omega}\left(\left(a_{n}, b_{n}\right),\left(a_{n+1}, b_{n+1}\right)\right) \leq s_{n}+d_{\Omega}\left(z_{n},\left(a_{n}, b_{n}\right)\right)$, and then

$$
d_{\Omega}\left(z_{n},\left(a_{n}, b_{n}\right)\right) \geq r_{n}-s_{n} \geq r_{n}-\log r_{n} .
$$

Standard hyperbolic trigonometry (see e.g. [9], p. 161) in $H_{n}$ gives

$$
\cosh \alpha_{n}=\frac{\cosh r_{n}+\cosh l_{n} \cosh l_{n+1}}{\sinh l_{n} \sinh l_{n+1}} \geq \frac{\frac{1}{2} e^{r_{n}}}{\frac{1}{2} e^{l_{n}} \frac{1}{2} e^{l_{n+1}}}+1=1+2 e^{r_{n}-l_{n}-l_{n+1}} .
$$

Then, we have

$$
\frac{1}{2} \sinh \alpha_{n} \geq \sinh \frac{\alpha_{n}}{2}=\sqrt{\frac{\cosh \alpha_{n}-1}{2}} \geq e^{\frac{1}{2}\left(r_{n}-l_{n}-l_{n+1}\right)} .
$$

Standard hyperbolic trigonometry for right-angled quadrilaterals gives

$$
\sinh d_{\Omega}\left(z_{n}, \gamma_{n}\right)=\sinh \alpha_{n} \cosh \left(l_{n+1}-s_{n}\right) \geq 2 e^{\frac{1}{2}\left(r_{n}-l_{n}-l_{n+1}\right)} \frac{1}{2} e^{l_{n+1}-\log r_{n}}=e^{\frac{1}{2}\left(r_{n}+l_{n+1}-l_{n}-2 \log r_{n}\right)} .
$$

Theorem 5.17. Let us consider a train $\Omega$ and a subsequence $\left\{n_{k}\right\}_{k}$ verifying either: 
(a) $\lim _{n \rightarrow \infty} l_{n}=\infty, \lim _{k \rightarrow \infty} r_{n_{k}}=\infty, l_{n_{k}} \leq l_{n_{k}+1}+c$ for every $k$ and $l_{n_{k}+1} \leq l_{n}+c$ for every $k$ and every $n \geq n_{k}+1$,

(b) $\lim _{k \rightarrow \infty} \bar{l}_{n_{k}+1}=\lim _{k \rightarrow \infty} r_{n_{k}}=\lim _{k \rightarrow \infty} r_{n_{k}+1}=\infty$ and $l_{n_{k}}, l_{n_{k}+2} \leq l_{n_{k}+1}+c$ for every $k$.

Then $\Omega$ is not hyperbolic.

Remark. The conclusion of Theorem 5.17 (with hypothesis $(a)$ ) also holds if we change condition " $l_{n_{k}} \leq$ $l_{n_{k}+1}+c$ for every $k$ " by "there exists an increasing function $F$ with $\lim _{t \rightarrow \infty} F(t)=\lim _{t \rightarrow \infty}(t-F(t))=\infty$ and $\lim _{k \rightarrow \infty}\left(r_{n_{k}}+l_{n_{k}+1}-l_{n_{k}}-2 F\left(\min \left\{l_{n_{k}+1}, r_{n_{k}}\right\}\right)\right)=\infty "$ (it is enough to change log by $F$ in the definition of $s_{n_{k}}$ in the proof below).

Proof. Let us assume hypothesis $(a)$. Consider $z_{n_{k}} \in \gamma_{n_{k}+1}^{+}$with $h\left(z_{n_{k}}\right)=l_{n_{k}+1}-s_{n_{k}}$, where $s_{n_{k}}:=$ $\log \left(\min \left\{l_{n_{k}+1}, r_{n_{k}}\right\}\right)$.

It is direct that $d_{\Omega}\left(z_{n_{k}},\left(a_{n_{k}+1}, b_{n_{k}+1}\right)\right)=s_{n_{k}}$ and $\lim _{k \rightarrow \infty} s_{n_{k}}=\infty$.

Lemma 5.16 implies the following facts:

$$
\begin{aligned}
d_{\Omega}\left(z_{n_{k}},\left(a_{0}, b_{0}\right)\right) & \geq l_{n_{k}+1}-\log l_{n_{k}+1} \longrightarrow \infty, \\
d_{\Omega}\left(z_{n_{k}},\left(a_{n_{k}}, b_{n_{k}}\right)\right) & \geq r_{n_{k}}-\log r_{n_{k}} \longrightarrow \infty, \\
d_{\Omega}\left(z_{n_{k}}, \gamma_{n_{k}}\right) & \geq \operatorname{Arcsinh} e^{\frac{1}{2}\left(r_{n_{k}}+l_{n_{k}+1}-l_{n_{k}}-2 \log r_{n_{k}}\right)} \geq \operatorname{Arcsinh} e^{\frac{1}{2}\left(r_{n_{k}}-c-2 \log r_{n_{k}}\right)} \longrightarrow \infty, \\
d_{\Omega}\left(z_{n_{k}}, \cup_{n=1}^{n_{k}-1}\left(a_{n}, b_{n}\right)\right) & \geq d_{\Omega}\left(z_{n_{k}}, \gamma_{n_{k}}\right) \longrightarrow \infty,
\end{aligned}
$$

if $k \rightarrow \infty$. Then $\lim _{k \rightarrow \infty} d_{\Omega}\left(z_{n_{k}}, \cup_{n=0}^{n_{k}+1}\left(a_{n}, b_{n}\right)\right)=\infty$. Since $l_{n_{k}+1} \leq l_{n}+c$ for every $k$ and every $n \geq n_{k}+1$, Lemma 5.15 gives that $\lim _{k \rightarrow \infty} d_{\Omega}\left(z_{n_{k}}, \mathbb{R}\right)=\infty$. Hence, $\Omega$ is not hyperbolic by Theorem 5.3.

Let us assume now hypothesis $(b)$. Consider $z_{n_{k}} \in \gamma_{n_{k}+1}^{+}$with $h\left(z_{n_{k}}\right)=l_{n_{k}+1}-s_{n_{k}}$, where $s_{n_{k}}:=$ $\log \left(\min \left\{l_{n_{k}+1}, r_{n_{k}}, r_{n_{k}+1}\right\}\right)$. The same argument of $(a)$ gives

$$
\begin{aligned}
d_{\Omega}\left(z_{n_{k}},\left(a_{n_{k}+1}, b_{n_{k}+1}\right)\right) & =s_{n_{k}}=\log \left(\min \left\{l_{n_{k}+1}, r_{n_{k}}, r_{n_{k}+1}\right\}\right) \longrightarrow \infty, \\
d_{\Omega}\left(z_{n_{k}},\left(a_{0}, b_{0}\right)\right) & \geq l_{n_{k}+1}-\log l_{n_{k}+1} \longrightarrow \infty, \\
d_{\Omega}\left(z_{n_{k}},\left(a_{n_{k}}, b_{n_{k}}\right)\right) & \geq r_{n_{k}}-\log r_{n_{k}} \longrightarrow \infty, \\
d_{\Omega}\left(z_{n_{k}}, \gamma_{n_{k}}\right) & \geq \operatorname{Arcsinh} e^{\frac{1}{2}\left(r_{n_{k}}+l_{n_{k}+1}-l_{n_{k}}-2 \log r_{n_{k}}\right)} \geq \operatorname{Arcsinh} e^{\frac{1}{2}\left(r_{n_{k}}-c-2 \log r_{n_{k}}\right)} \longrightarrow \infty, \\
d_{\Omega}\left(z_{n_{k}}, \cup_{n=1}^{n_{k}-1}\left(a_{n}, b_{n}\right)\right) & \geq d_{\Omega}\left(z_{n_{k}}, \gamma_{n_{k}}\right) \longrightarrow \infty,
\end{aligned}
$$

if $k \rightarrow \infty$. By symmetry ( since $r_{n_{k}+1}$ appears in the definition of $s_{n_{k}}$ ), we also have

$$
\begin{aligned}
d_{\Omega}\left(z_{n_{k}},\left(a_{n_{k}+2}, b_{n_{k}+2}\right)\right) & \geq r_{n_{k}+1}-\log r_{n_{k}+1} \longrightarrow \infty, \\
d_{\Omega}\left(z_{n_{k}}, \gamma_{n_{k}+2}\right) & \geq \operatorname{Arcsinh} e^{\frac{1}{2}\left(r_{n_{k}+1}+l_{n_{k}+1}-l_{n_{k}+2}-2 \log r_{n_{k}+1}\right)} \geq \operatorname{Arcsinh} e^{\frac{1}{2}\left(r_{n_{k}+1}-c-2 \log r_{n_{k}+1}\right)} \longrightarrow \infty, \\
d_{\Omega}\left(z_{n_{k}}, \cup_{n=n_{k}+3}^{\infty}\left(a_{n}, b_{n}\right)\right) & \geq d_{\Omega}\left(z_{n_{k}}, \gamma_{n_{k}+2}\right) \longrightarrow \infty,
\end{aligned}
$$

if $k \rightarrow \infty$. Then $\lim _{k \rightarrow \infty} d_{\Omega}\left(z_{n_{k}}, \mathbb{R}\right)=\infty$ and $\Omega$ is not hyperbolic by Theorem 5.3.

Corollary 5.18. Let us consider a train $\Omega$, with $\lim _{n \rightarrow \infty} l_{n}=\infty,\left\{l_{n}\right\}_{n}$ a non-decreasing sequence, and $\left\{r_{n}\right\}_{n}$ a non-bounded sequence. Then $\Omega$ is not hyperbolic.

Corollary 5.19. Let us consider a train $\Omega$, with $\lim _{n \rightarrow \infty} l_{n}=\lim _{n \rightarrow \infty} r_{n}=\infty$. Then $\Omega$ is not hyperbolic.

Proof. Since $\lim _{n \rightarrow \infty} l_{n}=\infty$, we can choose a subsequence $\left\{n_{k}\right\}_{k}$ with $l_{n_{k}+1} \leq l_{n}$ for every $k$ and every $n \geq n_{k}+1$.

If $l_{n_{k}} \leq l_{n_{k}+1}$ for infinitely many $k$ 's, part $(a)$ of Theorem 5.17 gives that $\Omega$ is not hyperbolic.

In other case, we have $l_{n_{k}}>l_{n_{k}+1}$ for every $k$ large enough. Then, given any $k$ large enough, it is clear that there exists $n_{k} \leq m_{k} \leq n_{k+1}$, with $l_{m_{k}}, l_{m_{k}+2} \leq l_{m_{k}+1}$. Consequently, part $(b)$ of Theorem 5.17 gives that $\Omega$ is not hyperbolic.

Sometimes it is convenient to split a train into "blocks" and to study locally the hyperbolicity in each of them. As we will see later, a valuable property of a block is that it is somehow "narrow". 
Definition 5.20. Given a train $\Omega$ and a subsequence $\left\{n_{k}\right\}_{k}$, we denote by $C_{n_{k}}$ the set $C_{n_{k}}:=\cup_{m=n_{k}}^{n_{k+1}-1} Y_{m}$. We say that $C_{n_{k}}$ is $c_{2}$-narrow if $d_{\Omega}\left(z, \mathbb{R} \cup \gamma_{n_{k}} \cup \gamma_{n_{k+1}}\right)=d_{\Omega}\left(z,\left(\mathbb{R} \cap C_{n_{k}}\right) \cup \gamma_{n_{k}} \cup \gamma_{n_{k+1}}\right) \leq c_{2}$ for every $z \in \cup_{m=n_{k}+1}^{n_{k+1}-1} \gamma_{m}$.

Next we study the case when (5.4) is only required for a subsequence.

Theorem 5.21. Let us consider a train $\Omega$ with $\lim _{n \rightarrow \infty} l_{n}=\infty$, and a subsequence $\left\{n_{k}\right\}_{k}$.

(a) Let us assume that $r_{n} \leq c_{1}+\left|l_{n}-l_{n+1}\right|$ and $l_{n} \geq l_{0}>0$ for every $n, l_{n_{1}} \leq l^{0}$, and $r_{n_{k}} \leq c_{1}$, $l_{n_{k}+1}+c_{1} \geq l_{n_{k+1}}$ for every $k$. If $C_{n_{k}}$ is $c_{2}$-narrow and

$$
\sum_{n=n_{k}+1}^{\infty} e^{-l_{n}} \leq c_{2} e^{-l_{n_{k}+1}}, \quad \text { for every } k,
$$

then $\Omega$ is $\delta$-hyperbolic, where $\delta$ is a constant which only depends on $c_{1}, c_{2}, l_{0}$ and $l^{0}$.

(b) Let us assume $l_{n}+c_{3} \geq l_{n_{k}}$ for every $k$ and every $n \geq n_{k}$. If $\Omega$ is hyperbolic, then there exists a constant $c_{4}$ such that $C_{n_{k}}$ is $c_{4}$-narrow and

$$
\sum_{n=n_{k}}^{\infty} e^{-l_{n}} \leq c_{4} e^{-l_{n_{k}}}, \quad \text { for every } k \text {. }
$$

\section{Remarks.}

1. A natural choice for $\left\{n_{k}\right\}_{k}$ is the set of indices corresponding to the largest non-decreasing subsequence of $\left\{l_{n}\right\}_{n}$. Observe that condition $l_{n_{k}+1}+c_{1} \geq l_{n_{k+1}}$ is natural in this context: if $l_{n_{k}+1}+c_{1}<$ $l_{n_{k+1}}$ for some $k$, then $n_{k}+1$ must belong to $\left\{n_{k}\right\}_{k}$.

2. Condition $l_{n} \geq l_{0}>0$ in $(a)$ is not restrictive at all since we have $\lim _{n \rightarrow \infty} l_{n}=\infty$.

Proof. In order to prove $(a)$, let us consider $z \in \cup_{n} \gamma_{n}$. If $z \in \gamma_{n_{k}}$ for some $k$, then Lemma 5.10 gives that there exists a constant $c_{5}$, which only depends on $c_{1}, c_{2}, l_{0}$ and $l^{0}$, such that $d_{\Omega}(z, \mathbb{R}) \leq c_{5}$. If $z \in \gamma_{n}$, with $n \notin\left\{n_{k}\right\}_{k}$, then $d_{\Omega}(z, \mathbb{R}) \leq c_{2}+c_{5}$, since $C_{n_{k}}$ is $c_{2}$-narrow for every $k$. Therefore, Theorem 5.3 gives the result.

If $\Omega$ is hyperbolic, then Theorem 5.3 gives that there exists a constant $c_{6}$ such that $d_{\Omega}(z, \mathbb{R}) \leq c_{6}$ for every $z \in \cup_{n} \gamma_{n}$. Hence $C_{n_{k}}$ is $c_{6}$-narrow for every $k$. Besides, Lemma 5.10 implies (5.6).

In order to obtain Lemma 5.23, which gives a criteria which assure that $C_{n_{k}}$ is $c_{1}$-narrow for every $k$, we need the following definition.

Definition 5.22. Given a subsequence $\left\{n_{k}\right\}_{k}$ in a train $\Omega$, we say that $C_{n_{k}}$ is c-admissible if there exist $n_{k} \leq n_{k}^{1} \leq n_{k}^{2} \leq n_{k}^{3} \leq n_{k}^{4} \leq n_{k+1}$ verifying $n_{k}^{1}-n_{k} \leq c, n_{k}^{3}-n_{k}^{2} \leq c, n_{k+1}-n_{k}^{4} \leq c$,

$$
\begin{array}{ll}
\sum_{k=n}^{n_{k}^{2}} e^{-l_{k}} \leq c e^{-l_{n}}, & \text { for every } n_{k}^{1}<n \leq n_{k}^{2}, \\
\sum_{k=n_{k}^{3}}^{n} e^{-l_{k}} \leq c e^{-l_{n}}, & \text { for every } n_{k}^{3} \leq n<n_{k}^{4} .
\end{array}
$$

Observe that $n_{k}^{j}$ and $n_{k}^{j+1}$ might coincide for some (or every) $j$.

Lemma 5.23. Let us consider a train $\Omega$ and a subsequence $\left\{n_{k}\right\}_{k}$. Let us assume that, for some $k, r_{n} \leq c_{1}$ for every $n_{k} \leq n<n_{k+1}$ and $C_{n_{k}}$ is $c_{2}$-admissible. Then there exists a constant $c_{3}$, which only depends on $c_{1}$ and $c_{2}$, such that $C_{n_{k}}$ is $c_{3}$-narrow.

Proof. Applying Lemma 5.9 at most $c_{2}$-times, we obtain that there exists a constant $c_{4}$, which only depends on $c_{1}$, such that $d_{\Omega}\left(z, \mathbb{R} \cup \gamma_{n_{k}}\right) \leq c_{2} c_{4}$ for every $z \in \cup_{n=n_{k}}^{n_{k}^{1}} \gamma_{n}$, and (by symmetry) $d_{\Omega}\left(z, \mathbb{R} \cup \gamma_{n_{k+1}}\right) \leq c_{2} c_{4}$ for every $z \in \cup_{n=n_{k}^{4}}^{n_{k+1}} \gamma_{n}$. We also have that $d_{\Omega}\left(z, \mathbb{R} \cup \gamma_{n_{k}^{2}}\right) \leq c_{2} c_{4}$ for every $z \in \cup_{n=n_{k}^{2}}^{n_{k}^{3}} \gamma_{n}$. 
By Corollary 5.11, there exists a constant $c_{5}$, which only depends on $c_{1}$ and $c_{2}$, such that $d_{\Omega}\left(z, \mathbb{R} \cup \gamma_{n_{k}^{1}}\right) \leq c_{5}$ for every $z \in \cup_{n=n_{k}^{1}}^{n_{k}^{2}} \gamma_{n}$, and (by symmetry) $d_{\Omega}\left(z, \mathbb{R} \cup \gamma_{n_{k}^{4}}\right) \leq c_{5}$ for every $z \in \cup_{n=n_{k}^{3}}^{n_{k}^{4}} \gamma_{n}$.

Hence, $d_{\Omega}\left(z, \mathbb{R} \cup \gamma_{n_{k}} \cup \gamma_{n_{k+1}}\right) \leq c_{3}:=2 c_{2} c_{4}+c_{5}$ for every $z \in \cup_{n=n_{k}}^{n_{k+1}} \gamma_{n}$, and $C_{n_{k}}$ is $c_{3}$-narrow.

The following result is a direct consequence of Theorem 5.21 and Lemma 5.23.

Theorem 5.24. Let us consider a train $\Omega$ and a subsequence $\left\{n_{k}\right\}_{k}$. Let us assume that $l_{n} \geq l_{0}$ and $r_{n} \leq c_{1}$ for every $n, l_{n_{1}} \leq l^{0}, l_{n_{k}+1}+c_{1} \geq l_{n_{k+1}}$ and $C_{n_{k}}$ is $c_{2}$-admissible for every $k$, and

$$
\sum_{n=n_{k}+1}^{\infty} e^{-l_{n}} \leq c_{2} e^{-l_{n_{k}+1}}, \quad \text { for every } k .
$$

Then $\Omega$ is $\delta$-hyperbolic, where $\delta$ is a constant which only depends on $c_{1}, c_{2}, l_{0}$ and $l^{0}$.

The hypotheses in Theorem 5.24 imply $\lim _{n \rightarrow \infty} l_{n}=\infty$. The ideas developed so far do allow us to deal now with results involving trains which do not hold condition $\lim _{n \rightarrow \infty} l_{n}=\infty$.

The first result uses the hypothesis $l_{n} \leq c$; it is a direct consequence of Theorem 5.3 (let us observe that in this result there is no condition on $\left.\left\{r_{n}\right\}_{n}\right)$.

Theorem 5.25. Let us consider a train $\Omega$, with $l_{n} \leq c$ for every $n$. Then $\Omega$ is $\delta$-hyperbolic, with $\delta$ a constant which only depends on $c$.

Proof. Fix $n$ and $z \in \gamma_{n}$. We have $d_{\Omega}(z, \mathbb{R}) \leq d_{\Omega}\left(z,\left(a_{0}, b_{0}\right) \cup\left(a_{n}, b_{n}\right)\right) \leq c / 2$. Hence, Theorem 5.3 gives the result.

The same argument proves the following result, in which only a subsequence of $\left\{l_{n}\right\}$ is required to be bounded.

Theorem 5.26. Let us consider a train $\Omega$ and a subsequence $\left\{n_{k}\right\}_{k}$. Let us assume that $r_{n} \leq c_{1}$ for every $n$ and $l_{n_{k}} \leq c_{1}$ for every $k$. Then $\Omega$ is $\delta$-hyperbolic if and only if $C_{n_{k}}$ is $c_{2}$-narrow for every $k$. Furthermore, if $C_{n_{k}}$ is $c_{2}$-narrow, then $\delta$ is a constant which only depends on $c_{1}$ and $c_{2}$.

Theorem 5.26 and Lemma 5.23 allows to deduce the following.

Theorem 5.27. Let us consider a train $\Omega$ and a subsequence $\left\{n_{k}\right\}_{k}$. Let us assume that $r_{n} \leq c$ for every $n$, and $l_{n_{k}} \leq c$ and $C_{n_{k}}$ is c-admissible for every $k$. Then $\Omega$ is $\delta$-hyperbolic, with $\delta$ a constant which only depends on $c$.

As a particular case, we obtain the next corollary.

Corollary 5.28. Let us consider a train $\Omega$ and a subsequence $\left\{n_{k}\right\}_{k}$. Let us assume that $r_{n} \leq c$ for every $n, l_{n_{k}} \leq c$ and $n_{k+1}-n_{k} \leq c$ for every $k$. Then $\Omega$ is $\delta$-hyperbolic, with $\delta$ a constant which only depends on $c$.

As we mentioned before, our results about trains may be somehow extended to a more general kind of spaces: generalized trains. From this point of view, Theorem 5.30 is the version for generalized trains of Theorem 5.3 for trains. This theorem together with Theorem 5.31 (applied to each $\left\{l_{n}^{k}\right\}_{n}$ ) provide criteria in order to decide about the hyperbolicity of generalized trains.

Definition 5.29. A generalized train is a Denjoy domain $\Omega \subset \mathbb{C}$ with $\Omega \cap \mathbb{R}=\cup_{k} \cup_{n=0}^{\infty}\left(a_{n}^{k}, b_{n}^{k}\right)$, such that $\sup _{n} b_{n}^{k} \leq \inf _{n} a_{n}^{k+1}$ for every $k$ or $\sup _{n} b_{n}^{k+1} \leq \inf _{n} a_{n}^{k}$ for every $k$, and for each $k$ we have either $b_{n}^{k} \leq a_{n+1}^{k}$ for every $n$ or $b_{n+1}^{k} \leq a_{n}^{k}$ for every $n$.

We denote by $\gamma_{n}^{k}$ the simple closed geodesic which just intersects $\mathbb{R}$ in $\left(a_{0}^{k}, b_{0}^{k}\right)$ and $\left(a_{n}^{k}, b_{n}^{k}\right)$. A generalized train is called $c$-controlled if $d_{\Omega}(z, \mathbb{R}) \leq c$, for every $z \in \cup_{n, k} \gamma_{n}^{k}$.

Remark. The index $k$ belongs either to $\mathbb{N}$ or to a finite set. 
Theorem 5.30. Let $\Omega$ be a generalized train. Then $\Omega$ is $\delta$-hyperbolic if and only if there exists a constant $c$ such that $\Omega$ is c-controlled, and for every $j \neq k$ there exist some $n, m$ and geodesics $g_{j k}$ joining $\left(a_{n}^{j}, b_{n}^{j}\right)$ and $\left(a_{m}^{k}, b_{m}^{k}\right)$ such that $d_{\Omega}(z, \mathbb{R}) \leq c$, for every $z \in \cup_{j \neq k} g_{j k}$.

Furthermore, if $\Omega$ is c-controlled and if $d_{\Omega}(z, \mathbb{R}) \leq c$ for every $z \in \cup_{j \neq k} g_{j k}$, then $\delta$ is a constant which only depends on $c$. If $\Omega$ is $\delta$-hyperbolic, then $c$ is a constant which only depends on $\delta$.

Proof. If $\Omega$ is $\delta$-hyperbolic, then Theorem 5.1 gives directly this implication.

In order to see the other implication, let us consider a geodesic $\alpha$ joining whatever two intervals $\left(a_{r}^{j}, b_{r}^{j}\right)$, $\left(a_{s}^{k}, b_{s}^{k}\right) \subset \Omega$. By Theorem 5.1, it is sufficient to prove that there exists a constant $c_{1}$, which only depends on $c$, such that $d_{\Omega}(z, \mathbb{R}) \leq c_{1}$ for every $z \in \alpha$. We can assume that $j \neq k$, since the case $j=k$ is easier.

Let us consider $z \in \alpha$. By symmetry, we can assume that $z \in \alpha^{+}$. By hypothesis, there exist a geodesic $g_{j k}$ joining $\left(a_{n}^{j}, b_{n}^{j}\right)$ and $\left(a_{m}^{k}, b_{m}^{k}\right)$ for some $n, m$, such that $d_{\Omega}(w, \mathbb{R}) \leq c$ for every $w \in g_{j k}$. Without loss of generality we can assume also that $g_{j k} \subset \Omega^{+}$. Now, we are going to consider the geodesics $\alpha_{r n} \subset \Omega^{+}$ which join $\left(a_{r}^{j}, b_{r}^{j}\right)$ with $\left(a_{n}^{j}, b_{n}^{j}\right)$ and $\alpha_{s m} \subset \Omega^{+}$joining $\left(a_{s}^{k}, b_{s}^{k}\right)$ with $\left(a_{m}^{k}, b_{m}^{k}\right)$. Corollary 5.4 gives that $d_{\Omega}(w, \mathbb{R}) \leq c+4 \log (1+\sqrt{2})$ for every $w \in \alpha_{r n} \cup \alpha_{s m}$.

Let us define the following geodesics: $\beta_{r}^{j} \subset\left(a_{r}^{j}, b_{r}^{j}\right)$ joining the end points of $\alpha$ and $\alpha_{r n}$ which belong to $\left(a_{r}^{j}, b_{r}^{j}\right), \beta_{n}^{j} \subset\left(a_{n}^{j}, b_{n}^{j}\right)$ joining the end points of $\alpha_{r n}$ and $g_{j k}$ which belong to $\left(a_{n}^{j}, b_{n}^{j}\right), \beta_{s}^{k} \subset\left(a_{s}^{k}, b_{s}^{k}\right)$ joining the end points of $\alpha$ and $\alpha_{s m}$ which belong to $\left(a_{s}^{k}, b_{s}^{k}\right)$ and $\beta_{m}^{k} \subset\left(a_{m}^{k}, b_{m}^{k}\right)$ joining the end points of $\alpha_{s m}$ and $g_{j k}$ which belong to $\left(a_{m}^{k}, b_{m}^{k}\right)$.

So, we have obtained a geodesic polygon $Q \subset \Omega^{+}$with at most eight sides; $Q$ is $6 \log (1+\sqrt{2})$-thin, since $\Omega^{+}$is isometric to a geodesically convex subset of the unit disk. Let us observe that the geodesic $\alpha$ is one of the sides of $Q$. Let us denote by $A$ the union of the other sides of the polygon. Then, there exists $w \in A$ with $d_{\Omega}(z, w) \leq 6 \log (1+\sqrt{2})$, and consequently $d_{\Omega}(z, \mathbb{R}) \leq d_{\Omega}(z, w)+d_{\Omega}(w, \mathbb{R}) \leq c_{1}:=10 \log (1+\sqrt{2})+c$.

We obtain directly the following result.

Theorem 5.31. Let $\Omega$ be a generalized train with $k$ belonging to a finite set. Then $\Omega$ is $\delta$-hyperbolic if and only if $\Omega$ is c-controlled.

Proof. If $\Omega$ is $\delta$-hyperbolic, then $\Omega$ is $c$-controlled, by Theorem 5.30 .

Let us assume now that $\Omega$ is $c$-controlled. For each $j \neq k$, choose geodesics $g_{j k}$ joining $\left(a_{0}^{j}, b_{0}^{j}\right)$ and $\left(a_{0}^{k}, b_{0}^{k}\right)$. Then $d_{\Omega}(z, \mathbb{R}) \leq \max _{j \neq k} L_{\Omega}\left(g_{j k}\right) / 2$, for every $z \in \cup_{j \neq k} g_{j k}$, and Theorem 5.30 implies the result.

Finally, a result which shows that hyperbolicity is stable under bounded perturbations of the lengths of the fundamental geodesics. Theorem 5.33 is particularly interesting since there are very few results on hyperbolic stability which do not involve quasi-isometries. We start with a technical lemma.

Lemma 5.32. Let us consider two trains $\Omega$ and $\Omega^{\prime}$ with $r_{n}=r_{n}^{\prime} \leq c_{1}$ for every $n, l_{n}^{\prime}=l_{n}+l_{0}$ if $l_{n}<l_{0}$ and $l_{n}^{\prime}=l_{n}$ if $l_{n} \geq l_{0}$. Then $\Omega$ is hyperbolic if and only if $\Omega^{\prime}$ is hyperbolic.

Furthermore, if $\Omega$ is $\delta$-hyperbolic, then $\Omega^{\prime}$ is $\delta^{\prime}$-hyperbolic, with $\delta^{\prime}$ a constant which only depends on $\delta, c_{1}$ and $l_{0}$; if $\Omega^{\prime}$ is $\delta^{\prime}$-hyperbolic, then $\Omega$ is $\delta$-hyperbolic, with $\delta$ a constant which only depends on $\delta^{\prime}, c_{1}$ and $l_{0}$.

Remark. $l_{n}^{\prime}$ and $r_{n}^{\prime}$ denote the lengths of the fundamental geodesics in $\Omega^{\prime}$.

Proof. To start with, let us suppose that $\Omega$ is $\delta$-hyperbolic and let us prove that $\Omega^{\prime}$ is $\delta^{\prime}$-hyperbolic.

Let us choose $z^{\prime} \in \gamma_{r}^{\prime} \subset \Omega^{\prime}$, for some $r$. By symmetry, without loss of generality we can assume that $z^{\prime} \in\left(\gamma_{r}^{\prime}\right)^{+}$. Now, let us take $z \in \gamma_{r}^{+} \subset \Omega$ with $h(z)=h\left(z^{\prime}\right)$. (Notice that if there not exists such $z$, it is because $d_{\Omega^{\prime}}\left(z^{\prime}, \mathbb{R}\right) \leq l_{0}$.)

Since $\Omega$ is $\delta$-hyperbolic, by Theorem 5.3 , there exists a constant $c_{2}$, which only depends on $\delta$, such that $d_{\Omega}(z, \mathbb{R})=d_{\Omega}(z, p(z)) \leq c_{2}$.

There are two possibilities:

(1) If $p(z) \in \gamma_{r}$, then there exists $z^{*} \in \gamma_{r}^{\prime} \cap \mathbb{R}$ with $d_{\Omega^{\prime}}\left(z^{\prime}, \mathbb{R}\right) \leq d_{\Omega^{\prime}}\left(z^{\prime}, z^{*}\right) \leq d_{\Omega}(z, p(z))+l_{0} \leq c_{2}+l_{0}$. 
(2) If $p(z) \notin \gamma_{r}$, we distinguish two cases.

If $l_{r}<l_{0}$, then $l_{r}^{\prime}=l_{r}+l_{0}<2 l_{0}$ and $d_{\Omega^{\prime}}\left(z^{\prime}, \mathbb{R}\right)<l_{0}$.

If $l_{r} \geq l_{0}$, let us denote by $g$ the geodesic joining $z$ and $p(z)$ such that $d_{\Omega}(z, \mathbb{R})=L_{\Omega}(g)$. Let us assume that $p(z) \in \cup_{n=1}^{r-1}\left(a_{n}, b_{n}\right)$ (if $p(z) \in \cup_{n=r+1}^{\infty}\left(a_{n}, b_{n}\right)$ the argument is symmetric). If $p(z) \in \gamma_{s}$ and $l_{n} \geq l_{0}$ for every $s \leq n \leq r$, then $l_{n}^{\prime}=l_{n}$ for every $s \leq n \leq r$ and $\cup_{n=s}^{r-1} Y_{n}$ is isometric to $\cup_{n=s}^{r-1} Y_{n}^{\prime}$, and then $d_{\Omega^{\prime}}\left(z^{\prime}, \mathbb{R}\right) \leq d_{\Omega}(z, p(z)) \leq c_{2}$. If $p(z) \in \gamma_{s}$ and $l_{n}<l_{0}$ for some $s \leq n \leq r$, let us define $m:=\max \left\{n<r: l_{n}<l_{0}\right\}, x:=g \cap \gamma_{m+1}$ and $d:=d_{\Omega}(z, x) \leq c_{2}$. Now, let us choose $x^{\prime} \in \gamma_{m+1}^{\prime}$ such that $h(x)=h\left(x^{\prime}\right)$ and let us call $d^{\prime}:=d_{\Omega^{\prime}}\left(z^{\prime}, x^{\prime}\right)$. Notice that $d=d^{\prime} \leq c_{2}$, since $\cup_{n=m+1}^{r-1} Y_{n}$ is isometric to $\cup_{n=m+1}^{r-1} Y_{n}^{\prime}$. Observe that the geodesic hexagon $H_{m}^{\prime}$ is $4 \log (1+\sqrt{2})$-thin, and therefore, $d_{\Omega^{\prime}}\left(x^{\prime}, \mathbb{R}\right) \leq 4 \log (1+\sqrt{2})+c_{1} / 2+l_{0}$ (recall that $r_{m}^{\prime} \leq c_{1}$ and $\left.l_{m}^{\prime}<2 l_{0}\right)$.

It means that

$$
d_{\Omega^{\prime}}\left(z^{\prime}, \mathbb{R}\right) \leq d_{\Omega^{\prime}}\left(z^{\prime}, x^{\prime}\right)+d_{\Omega^{\prime}}\left(x^{\prime}, \mathbb{R}\right) \leq c_{2}+4 \log (1+\sqrt{2})+c_{1} / 2+l_{0} .
$$

Consequently, $\Omega^{\prime}$ is $\delta^{\prime}$-hyperbolic, with $\delta^{\prime}$ a constant which only depends on $\delta, c_{1}$ and $l_{0}$, by Theorem 5.3 .

In order to prove that $\Omega^{\prime} \delta^{\prime}$-hyperbolic implies $\Omega \delta$-hyperbolic, we can follow a similar argument.

Theorem 5.33. Let us consider two trains $\Omega, \Omega^{\prime}$ and two constants $c_{1}, c_{2}$ such that $r_{n}, r_{n}^{\prime} \leq c_{1}$, and $\left|l_{n}^{\prime}-l_{n}\right| \leq c_{2}$. Then $\Omega$ is hyperbolic if and only if $\Omega^{\prime}$ is hyperbolic.

Furthermore, if $\Omega$ is $\delta$-hyperbolic, then $\Omega^{\prime}$ is $\delta^{\prime}$-hyperbolic, with $\delta^{\prime}$ a constant which only depends on $\delta, c_{1}$ and $c_{2}$.

Remark. Observe that in many cases $\Omega$ and $\Omega^{\prime}$ are not quasi-isometric (for example, if there exists a subsequence $\left\{n_{k}\right\}_{k}$ with $\lim _{k \rightarrow \infty} l_{n_{k}}=0$ and $l_{n_{k}}^{\prime} \geq c>0$ ).

Proof. By symmetry, it is sufficient to prove that if $\Omega$ is $\delta$-hyperbolic, then $\Omega^{\prime}$ is $\delta^{\prime}$-hyperbolic, with $\delta^{\prime}$ a constant which only depends on $\delta, c_{1}$ and $c_{2}$. Therefore, let us assume that $\Omega$ is $\delta$-hyperbolic.

By Lemma 5.32 we can assume that $l_{n}^{\prime}, l_{n} \geq 1$ for every $n$.

Given any point $z^{\prime} \in \gamma_{k}^{\prime}$, by Theorem 5.3 it is sufficient to prove that there exists a constant $c_{3}$, which only depends on $\delta, c_{1}$ and $c_{2}$, such that $d_{\Omega^{\prime}}\left(z^{\prime}, \mathbb{R}\right) \leq c_{3}$.

By symmetry, without loss of generality we can assume that $z^{\prime} \in\left(\gamma_{k}^{\prime}\right)^{+}$. Now, let us take $z \in \gamma_{k}^{+} \subset \Omega$ with $h(z)=h\left(z^{\prime}\right)$. (Notice that if there not exists such $z$, it is because $d_{\Omega^{\prime}}\left(z^{\prime}, \mathbb{R}\right) \leq c_{2}$.) Since $\Omega$ is $\delta$-hyperbolic, then $d_{\Omega}(z, \mathbb{R}) \leq c_{4}$, for some $c_{4}$, which only depends on $\delta$, by Theorem 5.3 .

There are two possibilities:

If $p(z) \in \gamma_{k}$, then there exists $z^{*} \in \gamma_{k}^{\prime} \cap \mathbb{R}$ with $d_{\Omega^{\prime}}\left(z^{\prime}, \mathbb{R}\right) \leq d_{\Omega^{\prime}}\left(z^{\prime}, z^{*}\right) \leq d_{\Omega}(z, p(z))+c_{2} \leq c_{2}+c_{4}$.

If $p(z) \notin \gamma_{k}$, then $p(z) \in\left(a_{m}, b_{m}\right)$, with $m \neq 0, k$. By symmetry we can assume that $0<m<k$. Let us denote by $g$ the geodesic joining $z$ and $p(z)$ such that $d_{\Omega}(z, \mathbb{R})=d_{\Omega}\left(z,\left(a_{m}, b_{m}\right)\right)=L_{\Omega}(g)$. Let us denote by $x$ the point $x:=g \cap \gamma_{m+1}$; we have $d:=d_{\Omega}(z, x) \leq c_{4}$ since $d_{\Omega}(z, \mathbb{R})=d_{\Omega}(z, x)+d_{\Omega}(x, \mathbb{R}) \leq c_{4}$.

We take $x^{\prime} \in\left(\gamma_{m+1}^{\prime}\right)^{+}$with $h\left(x^{\prime}\right)=\min \left\{h(x), l_{m+1}^{\prime}\right\}$. By the triangle inequality, $d_{\Omega^{\prime}}\left(z^{\prime}, \mathbb{R}\right) \leq d_{\Omega^{\prime}}\left(z^{\prime}, x^{\prime}\right)+$ $d_{\Omega^{\prime}}\left(x^{\prime}, \mathbb{R}\right)$. Now, let us try to get an upper bound for $d^{\prime}:=d_{\Omega^{\prime}}\left(z^{\prime}, x^{\prime}\right)$.

Since $l_{n}^{\prime}, l_{n} \geq 1$ for every $n$, by Lemma 5.5 we know that there exists a constant $c_{5}$, which only depends on $c_{1}$, such that for any $n$,

$$
\begin{aligned}
& e^{-l_{n}}+e^{-l_{n+1}} \leq \alpha_{n} \leq c_{5}\left(e^{-l_{n}}+e^{-l_{n+1}}\right), \\
& e^{-l_{n}^{\prime}}+e^{-l_{n+1}^{\prime}} \leq \alpha_{n}^{\prime} \leq c_{5}\left(e^{-l_{n}^{\prime}}+e^{-l_{n+1}^{\prime}}\right) .
\end{aligned}
$$

In order to simplify the notation we are going to define $B$ and $B^{\prime}$ as

$$
B:=\sum_{n=m+1}^{k-1}\left(e^{-l_{n}}+e^{-l_{n+1}}\right), \quad B^{\prime}:=\sum_{n=m+1}^{k-1}\left(e^{-l_{n}^{\prime}}+e^{-l_{n+1}^{\prime}}\right) .
$$


It is clear that $e^{-c_{2}} \leq B / B^{\prime} \leq e^{c_{2}}$. By hyperbolic trigonometry,

$$
\begin{aligned}
\cosh d & =\cosh \left(\sum_{n=m+1}^{k-1} \alpha_{n}\right) \cosh h(z) \cosh h(x)-\sinh h(z) \sinh h(x) \\
& \geq \cosh B \cosh h(z) \cosh h(x)-\sinh h(z) \sinh h(x) \geq(\cosh B-1) \cosh h(z) \cosh h(x) .
\end{aligned}
$$

Let us assume that $h\left(x^{\prime}\right)=h(x)$; then we obtain

$$
\begin{aligned}
\cosh d^{\prime} & =\cosh \left(\sum_{n=m+1}^{k-1} \alpha_{n}^{\prime}\right) \cosh h(z) \cosh h(x)-\sinh h(z) \sinh h(x) \\
& \leq \cosh \left(c_{5} B^{\prime}\right) \cosh h(z) \cosh h(x)-\sinh h(z) \sinh h(x) \\
& \leq \cosh \left(c_{5} e^{c_{2}} B\right) \cosh h(z) \cosh h(x)-\sinh h(z) \sinh h(x) \\
& =\frac{\cosh \left(c_{5} e^{c_{2}} B\right)-1}{\cosh B-1}(\cosh B-1) \cosh h(z) \cosh h(x)+\cosh h(z) \cosh h(x)-\sinh h(z) \sinh h(x) \\
& \leq \frac{\cosh \left(c_{5} e^{c_{2}} B\right)-1}{\cosh B-1} \cosh d+\cosh (h(z)-h(x))
\end{aligned}
$$

It is clear that $B \leq \sum_{n=m+1}^{k-1} \alpha_{n}=d_{\Omega}\left(z_{0}, x_{0}\right) \leq d_{\Omega}(z, x) \leq c_{4}$. Since $c_{5} e^{c_{2}}>1$, the function $\frac{\cosh \left(c_{5} e^{c_{2}} B\right)-1}{\cosh B-1}$ is increasing in $B$; hence,

$$
\frac{\cosh \left(c_{5} e^{c_{2}} B\right)-1}{\cosh B-1} \leq \frac{\cosh \left(c_{5} e^{c_{2}} c_{4}\right)-1}{\cosh c_{4}-1} .
$$

Besides, $|h(z)-h(x)| \leq d_{\Omega}(z, x) \leq c_{4}$ by Lemma 5.7. Then

$$
d^{\prime} \leq \operatorname{Arccosh}\left(\frac{\cosh \left(c_{5} e^{c_{2}} B\right)-1}{\cosh B-1} \cosh c_{4}+\cosh c_{4}\right) .
$$

If $h\left(x^{\prime}\right)<h(x)$, then $h\left(x^{\prime}\right)=l_{m+1}^{\prime}$ and $h(x)-h\left(x^{\prime}\right)=h(x)-l_{m+1}^{\prime} \leq l_{m+1}-l_{m+1}^{\prime} \leq c_{2}$. Hence, $\left|h(z)-h\left(x^{\prime}\right)\right| \leq$ $|h(z)-h(x)|+h(x)-h\left(x^{\prime}\right) \leq c_{4}+c_{2}$. With the same computations we obtain

$$
\begin{aligned}
\cosh d^{\prime} & \leq \frac{\cosh \left(c_{5} e^{c_{2}} B\right)-1}{\cosh B-1} \cosh d+\cosh \left(h(z)-h\left(x^{\prime}\right)\right), \\
d^{\prime} & \leq c_{6}:=\operatorname{Arccosh}\left(\frac{\cosh \left(c_{5} e^{c_{2}} B\right)-1}{\cosh B-1} \cosh c_{4}+\cosh \left(c_{2}+c_{4}\right)\right) .
\end{aligned}
$$

Now we consider $d_{\Omega^{\prime}}\left(x^{\prime}, \mathbb{R}\right)$ (recall that $\left.x^{\prime} \in \gamma_{m+1}^{\prime}\right)$.

We can assume that $h\left(x^{\prime}\right)=h(x)$, since if $h\left(x^{\prime}\right)<h(x)$, then $x^{\prime} \in \mathbb{R}$ and $d_{\Omega^{\prime}}\left(z^{\prime}, \mathbb{R}\right) \leq d_{\Omega^{\prime}}\left(z^{\prime}, x^{\prime}\right) \leq c_{6}$.

There are two possibilities.

If $h\left(x^{\prime}\right) \geq l_{m}^{\prime}$, then by Lemma 5.8 there exists a constant $c_{7}$, which only depends on $c_{1}$, such that $d_{\Omega^{\prime}}\left(x^{\prime}, \mathbb{R}\right) \leq c_{7}$.

If $h\left(x^{\prime}\right)<l_{m}^{\prime}$, then $h(x)=h\left(x^{\prime}\right)<l_{m}+c_{2}$. If $h(x) \geq l_{m}$, then $h\left(x^{\prime}\right) \geq l_{m}^{\prime}-c_{2} ;$ so $d_{\Omega^{\prime}}\left(x^{\prime}, \mathbb{R}\right) \leq c_{2}+c_{7}$. If $h(x)<l_{m}$, we have that $l_{m}-h(x) \leq h(p(z))-h(x) \leq d_{\Omega}(x, \mathbb{R}) \leq c_{4}$ and it is easy to check that $l_{m}^{\prime}-h\left(x^{\prime}\right) \leq l_{m}+c_{2}-h(x) \leq c_{2}+c_{4} ;$ so $d_{\Omega^{\prime}}\left(x^{\prime}, \mathbb{R}\right) \leq c_{2}+c_{4}+c_{7}$.

Therefore $d_{\Omega^{\prime}}\left(z^{\prime}, \mathbb{R}\right) \leq d_{\Omega^{\prime}}\left(z^{\prime}, x^{\prime}\right)+d_{\Omega^{\prime}}\left(x^{\prime}, \mathbb{R}\right) \leq c_{3}:=c_{6}+c_{2}+c_{4}+c_{7}$.

Consequently $\Omega^{\prime}$ is $\delta^{\prime}$-hyperbolic with $\delta^{\prime}$ a constant which only depends on $\delta, c_{1}$ and $c_{2}$.

\section{REFERENCES}

[1] Ahlfors, L., Sario, L., Riemann surfaces. Princeton Univ. Press, Princeton, New Jersey, 1960.

[2] Aikawa, H., Positive harmonic functions of finite order in a Denjoy type domain, Proc. Amer. Math. Soc. 131 (2003), 3873-3881. 
[3] Alvarez, V., Pestana, D., Rodríguez, J. M., Isoperimetric inequalities in Riemann surfaces of infinite type. Rev. Mat. Iberoamericana 15 (1999), 353-427.

[4] Alvarez, V., Rodríguez, J.M., Yakubovich, V.A., Subadditivity of p-harmonic "measure" on graphs, Michigan Mathematical Journal 49 (2001), 47-64.

[5] Anderson, J. W., Hyperbolic Geometry. Springer, London, 1999.

[6] Balogh, Z. M., Buckley, S. M., Geometric characterizations of Gromov hyperbolicity, Invent. Math. 153 (2003), $261-301$.

[7] Basmajian, A., Constructing pair of pants, Ann. Acad. Sci. Fenn. Series AI 15 (1990), 65-74.

[8] Basmajian, A., Hyperbolic structures for surfaces of infinite type, Trans. Amer. Math. Soc. 336 (1993), $421-444$.

[9] Bers, L., An Inequality for Riemann Surfaces. Differential Geometry and Complex Analysis. H. E. Rauch Memorial Volume. Springer-Verlag. 1985.

[10] Bishop, R., O’Neill, B., Manifolds of negative curvature, Trans. Amer. Math. Soc. 145 (1969), 1-49.

[11] Bonk, M., Quasi-geodesics segments and Gromov hyperbolic spaces, Geometriae Dedicata 62 (1996), 281-298.

[12] Bonk, M., Heinonen, J., Koskela, P., Uniformizing Gromov hyperbolic spaces. Astérisque No. 270 (2001).

[13] Buser, P., Geometry and Spectra of Compact Riemann Surfaces. Birkhäuser, Boston, 1992.

[14] Cantón, A., Fernández, J. L., Pestana, D., Rodríguez, J. M., On harmonic functions on trees, Potential Analysis 15 (2001), 199-244.

[15] Chavel, I., Eigenvalues in Riemannian Geometry. Academic Press, New York, 1984.

[16] Coornaert, M., Delzant, T., Papadopoulos, A. Notes sur les groups hyperboliques de Gromov. I.R.M.A., Strasbourg, 1989.

[17] Eberlein, P., Surfaces of nonpositive curvature. Memoirs of the American Mathematical Society, Volume 20 , Number 218. Providence, Rhode Island. 1979.

[18] Fenchel, W., Elementary Geometry in Hyperbolic Space. Walter de Gruyter, Berlin-New York, 1989.

[19] Fernández, J. L., Rodríguez, J. M., The exponent of convergence of Riemann surfaces. Bass Riemann surfaces. Ann. Acad. Sci. Fenn. Series AI 15 (1990), 165-183.

[20] Fernández, J. L., Rodríguez, J. M., Area growth and Green's function of Riemann surfaces, Arkiv för matematik 30 (1992), 83-92.

[21] Garnett, J., Jones, P., The Corona theorem for Denjoy domains, Acta Math. 155 (1985), 27-40.

[22] Ghys, E., de la Harpe, P., Sur les Groupes Hyperboliques d'après Mikhael Gromov. Progress in Mathematics, Volume 83. Birkhäuser. 1990.

[23] González, M. J., An estimate on the distortion of the logarithmic capacity, Proc. Amer. Math. Soc. 126 (1998), $1429-1431$.

[24] Haas, A., Dirichlet points, Garnett points, and infinite ends of hyperbolic surfaces I, Ann. Acad. Sci. Fenn. Series AI 21 (1996), 3-29.

[25] Holopainen, I., Soardi, P. M., p-harmonic functions on graphs and manifolds, Manuscripta Math. 94 (1997), 95-110.

[26] Kanai, M., Rough isometries and combinatorial approximations of geometries of non-compact Riemannian manifolds, $J$. Math. Soc. Japan 37 (1985), 391-413.

[27] Kanai, M., Rough isometries and the parabolicity of Riemannian manifolds, J. Math. Soc. Japan 38 (1986), $227-238$.

[28] Ortega, J., Seip, K., Harmonic measure and uniform densities. Preprint.

[29] Paulin, F., On the critical exponent of a discrete group of hyperbolic isometries, Diff. Geom. Appl. 7 (1997), 231-236.

[30] Portilla, A., Rodríguez, J. M., Tourís, E., Gromov hyperbolicity through decomposition of metric spaces II, J. Geom. Anal. 14 (2004), 123-149.

[31] Portilla, A., Rodríguez, J. M., Tourís, E., The topology of balls and Gromov hyperbolicity of Riemann surfaces. Diff. Geom. Appl. 21 (2004), 317-335.

[32] Portilla, A., Rodríguez, J. M., Tourís, E., The role of funnels and punctures in the Gromov hyperbolicity of Riemann surfaces. To appear in Proceedings Edinburgh Mathematical Society.

[33] Randol, B., Cylinders in Riemann surfaces, Comment. Math. Helv. 54 (1979), 1-5.

[34] Shimizu, H., On discontinuous groups operating on the product of upper half-planes, Ann. of Math. 77 (1963), 33-71.

[35] Soardi, P. M., Rough isometries and Dirichlet finite harmonic functions on graphs, Proc. Amer. Math. Soc. 119 (1993), 1239-1248.

Ana Portilla, José M. Rodríguez, Eva Tourís

Departamento de Matemáticas

Escuela Politécnica Superior

Universidad Carlos III de Madrid

Avenida de la Universidad, 30

28911 Leganés (Madrid)

SPAIN
Venancio Alvarez

Departamento de Análisis Matemático

Facultad de Ciencias

Campus de Teatinos

29071 Málaga

SPAIN

E-mail address: apferrei@math.uc3m.es, jomaro@math.uc3m.es, etouris@math.uc3m.es, nancho@anamat.cie.uma.es 